\title{
SPACES OF FRACTIONS AND POSITIVE FUNCTIONALS
}

\author{
F.-H. VASILESCU
}

\begin{abstract}
We discuss integral representations and extensions of positive functionals in some spaces of fractions of continuous functions. As a consequence, numerical caracterizations for the solvability of power moment problems and the uniqueness of the solutions in unbounded sets are obtained.
\end{abstract}

\section{Introduction}

Let $\mathrm{K}$ be the real field $\mathrm{R}$ or the complex one $\mathrm{C}$. We consider vector spaces over the field $\mathrm{K}$, and $\mathrm{K}$-linear maps defined between such spaces. When no confusion is possible, the expression "K-linear" will be replaced by "linear".

Let $\Omega$ be a compact Hausdorff space and let $C_{\mathrm{K}}(\Omega)$ be the algebra of all $\mathrm{K}$-valued continuous functions on $\Omega$, endowed with the natural norm $\|f\|_{\infty}=$ $\sup _{\omega \in \Omega}|f(\omega)|, f \in C_{\mathrm{K}}(\Omega)$. It is well known that every positive linear functional on $C_{\mathrm{K}}(\Omega)$ has an integral representation. Specifically, if $\psi: C_{\mathrm{K}}(\Omega) \rightarrow \mathrm{K}$ is linear and positive, then there exists a uniquely determined positive measure $\mu$ on $\Omega$ such that $\psi(f)=\int_{\Omega} f d \mu, f \in C_{\mathrm{K}}(\Omega)$. As a matter of fact, if $\psi: C_{\mathrm{K}}(\Omega) \rightarrow \mathrm{K}$ is linear, then $\psi$ is positive if and only if $\psi$ is continuous and $\|\psi\|=\psi(1)$.

These features of positive linear functionals can be partially or totally recaptured in more general spaces, derived from the basic model $C_{\mathrm{K}}(\Omega)$.

Let $\mathscr{Q}$ be a family of nonnull positive elements of $C_{\mathrm{K}}(\Omega)$. We say that 2 is a multiplicative family if (i) $1 \in \mathscr{Q}$, (ii) $q^{\prime}, q^{\prime \prime} \in \mathscr{Q}$ implies $q^{\prime} q^{\prime \prime} \in \mathscr{Q}$, and (iii) if $q h=0$ for some $q \in \mathscr{Q}$ and $h \in C_{\mathrm{K}}(\Omega)$, then $h=0$.

Let $C_{\mathrm{K}}(\Omega) / \mathscr{Q}$ denote the algebra of fractions with numerators in $C_{\mathrm{K}}(\Omega)$, and with denominators in the multiplicative family $\mathscr{Q}$, which is a unital $\mathrm{K}$ algebra (see, for instance, [16] for details). This algebra has a natural involution $f \rightarrow \bar{f}$, induced by the natural involution of $C_{\mathrm{K}}(\Omega)$ (which is the identity for $\mathrm{K}=R$ ). 
To define a natural topological structure on $C_{K}(\Omega) / \mathscr{Q}$, we note that for every $f \in C_{\mathrm{K}}(\Omega) / \mathscr{Q}$ we can find a $q \in \mathscr{Q}$ such that $q f \in C_{\mathrm{K}}(\Omega)$. If

$$
C_{\mathrm{K}}(\Omega) / q=\left\{f \in C_{\mathrm{K}}(\Omega) / \mathscr{2} ; q f \in C_{\mathrm{K}}(\Omega)\right\},
$$

then we have $C_{\mathrm{K}}(\Omega) / \mathscr{Q}=\cup_{q \in \mathscr{2}} C_{\mathrm{K}}(\Omega) / q$. Setting $\|f\|_{\infty, q}=\|q f\|_{\infty}$ for each $f \in C_{\mathrm{K}}(\Omega) / q$, the pair $\left(C_{\mathrm{K}}(\Omega) / q,\|*\|_{\infty, q}\right)$ becomes a Banach space. For this reason, $C_{\mathrm{K}}(\Omega) / 2$ can be naturally regarded as an inductive limit of Banach spaces (see [11], Section V.2).

Elementary facts related to the theory of inductive limits of normed spaces, necessary for further development, are presented in the next section.

In each space $C_{\mathrm{K}}(\Omega) / q$ we have a positive cone $\left(C_{\mathrm{K}}(\Omega) / q\right)^{+}$consisting of those elements $f \in C_{\mathrm{K}}(\Omega) / q$ such that $q f \geq 0$ as a continuous function. We say that $f$ is positive if $f \in\left(C_{\mathrm{K}}(\Omega) / q\right)^{+}$.

The positive elements of the algebra $C_{K}(\Omega) / \mathscr{Q}$ are, by definition, the members of the cone $\left(C_{\mathrm{K}}(\Omega) / \mathscr{2}\right)^{+}$, consisting of all finite sums of positive elements from the cones $\left(C_{K}(\Omega) / q\right)^{+}$, with $q \in \mathscr{Q}$ arbitrary. The positivity of a linear map on $C_{\mathrm{K}}(\Omega) / \mathscr{Q}$ will be defined with respect to the positive cone $\left(C_{\mathrm{K}}(\Omega) / \mathscr{Q}\right)^{+}$.

The aim of this work is describe the positive functionals in the algebra of fractions $C_{\mathrm{K}}(\Omega) / \mathscr{Q}$, and to characterize those linear functionals on some subspaces of $C_{\mathrm{K}}(\Omega) / \mathscr{Q}$ which extend to positive ones. The main result (Theorem 3.7), whose proof uses a weak*-compactness argument inspired from [14], will be applied to characterize the solvability of the multidimensional power moment problem (in particular, the several variable Hamburger moment problem), in numerical terms (Theorems 4.1, 4.2, and 4.8). The uniqueness of the solution of the moment problem in unbounded closed sets is also characterized in numerical terms (see Theorem 4.5).

Let us briefly present our characterisation of the existence of a solution for the Hamburger moment problem in one variable. Let $\mu$ be a positive measure on the real line $\mathrm{R}$ such that the monomial $t^{k} \in L^{1}(\mu)$ for all integers $k \geq 0$ (i.e., the measure $\mu$ has moments of all orders). If $p$ is any polynomial of degree $d \geq 0$ then we clearly have

$$
\left|\int_{\mathrm{R}} p(t) d \mu(t)\right| \leq \sup _{t \in \mathrm{R}} \frac{|p(t)|}{\left(1+t^{2}\right)^{m}} \int_{\mathrm{R}}\left(1+t^{2}\right)^{m} d \mu(t),
$$

where the integer $m$ satisfies $d \leq 2 m$. These necessary conditions, which can be expressed only in terms of moments of $\mu$, are also sufficient, even for several variables. Moreover, the quantities $\sup _{t \in \mathrm{R}}|p(t)| /\left(1+t^{2}\right)^{m}$ are universal and calculable, at least in principle. In Remark 4.11, we present a method of computation of such sup-norms. 
The classical result of Haviland concerning the solvability of the moment problem (see [7]) can be obtained as a consequence of Theorem 4.2 (see Corollary 4.4). The characterization in terms of semi-norms stated in Proposition 4.6 gives a different insight to the moment problem in unbounded sets, with references to the determinacy in terms of density (see Proposition 4.7). All these results are in concordance with the corresponding assertions from [9] and [15]. Moment problems in one or several variables are extensively studied [1], [2], [6], [13] etc. Other descriptions of positive functionals on various spaces, using different methods, can be found in [12].

The author is indebted to E. Albrecht (Saarbrücken) and O. Demanze (Lille) for useful discussions on early versions of this work.

\section{Linear functionals on inductive limits}

In this section we present some auxiliary results, concerning the linear functionals defined on inductive limits of vector spaces (see, for instance, [11], Section V.2). We restrict ourselves to inductive limits of normed spaces.

Let $X$ be a vector space over the field $\mathrm{K}$. Following [11], the space $X$ is said to be an inductive limit (of normed spaces) if $X$ is spanned by a family of vector subspaces $\left(X_{\alpha}\right)_{\alpha \in A}$, such that each $X_{\alpha}$ is a normed space.

Unlike in [11], we use the notation $X=\sum_{\alpha \in A} X_{\alpha}$ to indicate that $X$ is the inductive limit of the family of normed subspaces $\left(X_{\alpha}\right)_{\alpha \in A}$.

Suppose that $X=\sum_{\alpha \in A} X_{\alpha}$. In this case, a neighborhood base of $X$ is given by the family of sets of the form

$$
V_{\rho}=\operatorname{co}\left(\bigcup_{\alpha \in A}\left\{x \in X_{\alpha} ;\|x\|_{\alpha} \leq \rho_{\alpha}\right\}\right),
$$

where $\|*\|_{\alpha}$ is the norm on $X_{\alpha}, \rho=\left(\rho_{\alpha}\right)_{\alpha \in A}$ is a family of positive numbers and "co" stands for the "convex hull". In fact, it is easily seen that each convex set $V_{\rho}$ is also balanced and absorbent.

For each set $V_{\rho} \subset X$ as above, we denote by $\sigma_{V_{\rho}}$ its gauge function (see [11]), which is a seminorm on $X$. Specifically,

$$
\begin{array}{r}
\sigma_{V_{\rho}}(x)=\inf _{F}\left\{\sum_{\alpha \in F} \lambda_{\alpha} ; x=\sum_{\alpha \in F} \lambda_{\alpha} x_{\alpha}, \lambda_{\alpha} \geq 0, x_{\alpha} \in X_{\alpha},\left\|x_{\alpha}\right\|_{\alpha} \leq \rho_{\alpha}, \alpha \in F\right\}, \\
x \in X,
\end{array}
$$

where $F$ runs the finite subsets of $A$. It is easily seen that

$$
\sigma_{V_{\rho}}(x)=\inf _{F}\left\{\sum_{\alpha \in F} \rho_{\alpha}^{-1}\left\|x_{\alpha}\right\|_{\alpha} ; x=\sum_{\alpha \in F} x_{\alpha}, x_{\alpha} \in X_{\alpha}, \alpha \in F\right\}, \quad x \in X,
$$


where $F$ runs the finite subsets of $A$.

In particular, $\sigma_{V_{\rho}}(x) \leq \rho_{\alpha}^{-1}\|x\|_{\alpha}$ for all $x \in X_{\alpha}$ and $\alpha \in A$.

A linear functional $\psi: X \rightarrow \mathrm{K}$ is said to be continuous if $\psi_{\alpha}=\psi \mid X_{\alpha}$ is continuous for all $\alpha \in A$.

We want to describe the continuity of a linear functional in terms of gauge functions. Some of these (elementary) results are possibly known. We preserve the notation from above.

Lemma 2.1. Let $\psi: X \rightarrow \mathrm{K}$ be linear.

(a) If $\psi$ is continuous, then $|\psi(x)| \leq \sigma_{V_{\rho}}(x), x \in X$, where $\rho_{\alpha}=\left\|\psi_{\alpha}\right\|^{-1}$ if $\psi_{\alpha} \neq 0$ and $\rho_{\alpha}>0$ is arbitrary if $\psi_{\alpha}=0$.

(b) Conversely, if $|\psi(x)| \leq \sigma_{V_{\rho}}(x), x \in X$, for a certain $V_{\rho}$, then $\left\|\psi_{\alpha}\right\| \leq$ $\rho_{\alpha}^{-1}$ for all $\alpha \in A$.

Proof. (a) If $x \in X$ and $\epsilon>0$ are given, then there exists a representation $x=\sum_{k \in K} \lambda_{k} x_{k}$, where $\lambda_{k} \geq 0, x_{k} \in X_{\alpha_{k}},\left\|x_{k}\right\|_{\alpha_{k}} \leq \rho_{\alpha_{k}}, k \in K, K \subset \mathbf{Z}_{+}$is finite and $\sum_{k \in K} \lambda_{k} \leq \sigma_{V_{\rho}}(x)+\epsilon$. Assuming, with no loss of generality, that $\psi_{\alpha_{k}} \neq 0$ for $k \in K$, we have:

$$
|\psi(x)| \leq \sum_{k \in K} \lambda_{k}\left|\psi_{\alpha_{k}}\left(x_{k}\right)\right| \leq \sum_{k \in K} \lambda_{k}\left\|\psi_{\alpha_{k}}\right\|\left\|x_{k}\right\|_{\alpha_{k}} \leq \sigma_{V_{\rho}}(x)+\epsilon .
$$

As $\epsilon>0$ is arbitrary, we obtain the estimate $|\psi(x)| \leq \sigma_{V_{\rho}}(x), x \in X$.

(b) Conversely, fix an $\alpha \in A$ such that $\psi_{\alpha} \neq 0$, and let $x \in X_{\alpha}, x \neq 0$. If $y=\|x\|_{\alpha}^{-1} \rho_{\alpha} x$, then $y \in V_{\rho}$, and so $|\psi(y)| \leq 1$, whence $|\psi(x)| \leq\|x\|_{\alpha} \rho_{\alpha}^{-1}$, implying $\left\|\psi_{\alpha}\right\| \leq \rho_{\alpha}^{-1}$.

Lemma 2.2. Let $Y \subset X$ be a vector subspace and let $\phi: Y \rightarrow \mathrm{K}$ be linear. Let also $Y_{\alpha}=Y \cap X_{\alpha}$ and $\phi_{\alpha}=\phi \mid Y_{\alpha}, \alpha \in A$. Suppose that $\phi_{\alpha}$ is continuous and $\neq 0$ for all $\alpha \in A$.

The functional $\phi$ has a continuous linear extension $\psi: X \rightarrow \mathrm{K}$ such that $\left\|\psi_{\alpha}\right\|=\left\|\phi_{\alpha}\right\|, \alpha \in A$, if and only if $|\phi(y)| \leq \sigma_{V_{\rho}}(y), y \in Y$, where $\rho_{\alpha}=\left\|\phi_{\alpha}\right\|^{-1}, \alpha \in A$.

Proof. If $\psi: X \rightarrow \mathrm{K}$ is a continuous extension of $\phi: Y \rightarrow \mathrm{K}$ such that $\left\|\psi_{\alpha}\right\|=\left\|\phi_{\alpha}\right\|, \alpha \in A$, then $|\psi(x)| \leq \sigma_{V_{\rho}}(x), x \in X$, where $\rho_{\alpha}=\left\|\psi_{\alpha}\right\|^{-1}=$ $\left\|\phi_{\alpha}\right\|^{-1}$, by Lemma 2.1(a). In particular, $|\phi(y)| \leq \sigma_{V_{\rho}}(y), y \in Y$.

Conversely, if $|\phi(y)| \leq \sigma_{V_{\rho}}(y), y \in Y$, the Hahn-Banach theorem implies the existence of an extension $\phi: Y \rightarrow \mathrm{K}$ such that $|\psi(x)| \leq \sigma_{V_{\rho}}(x), x \in X$. It follows from Lemma 2.1(b) that $\left\|\psi_{\alpha}\right\| \leq\left\|\phi_{\alpha}\right\|, \alpha \in A$. But $\psi_{\alpha}$ is an extension of $\phi_{\alpha}$, and so $\left\|\psi_{\alpha}\right\|=\left\|\phi_{\alpha}\right\|$ for all $\alpha \in A$.

Remark 2.3. We keep the notation from Lemma 2.2. Let $Z=\{x \in$ $\left.X ; \sigma_{V_{\rho}}(x)=0\right\}$, which is a vector subspace of $X$. Then the quotient $X / Z$ is a normed space, whose norm is given by $\|x+Z\|=\sigma_{V_{\rho}}(x), x \in X$. 
Suppose the functional $\phi: Y \rightarrow \mathrm{K}$ continuous. Then $\phi$ induces a functional $\Phi:(Y+Z) / Z \rightarrow \mathrm{K}$ by the formula $\Phi(y+Z)=\psi(y), y \in Y$. This definition is correct, because the trivial extension $\tilde{\phi}(y+z)=\phi(y), y \in Y, z \in Z$, is well defined and continuous on $Y+Z$, via the equality $\sigma_{V_{\rho}}(y+z)=\sigma_{V_{\rho}}(y)$.

The functional $\phi: Y \rightarrow \mathrm{K}$ extends uniquely to a continuous linear functional $\psi: X \rightarrow \mathrm{K}$ if and only if the subspace $(Y+Z) / Z$ is dense in $X / Z$. Indeed, if $(Y+Z) / Z$ is dense in $X / Z$, then $\Phi$ has a unique continuous extension $\Psi: X / Z \rightarrow \mathrm{K}$. The functional $\psi: X \rightarrow \mathrm{K}$, given by $\psi(x)=\Psi(x+Z)$, $x \in X$, is unique because any other continuous extension $\psi^{\prime}: X \rightarrow \mathrm{K}$ of $\phi$ gives rise to an extension $\Psi^{\prime}: X / Z \rightarrow \mathrm{K}$ of $\Phi$, which must be equal to $\Psi$. This implies the equality $\psi^{\prime}=\psi$.

Conversely, if $(Y+Z) / Z$ is not dense in $X / Z$, we can find a nonnull continuous linear functional $\Gamma: X / Z \rightarrow \mathrm{K}$ which is null on $(Y+Z) / Z$. If $\gamma: Y \rightarrow \mathrm{K}$ is the functional induced by $\Gamma$, then for any extension $\psi$ of $\phi$, the functional $\psi+\gamma$ is another extension, different from $\psi$.

REMARK 2.4. We recall some well known facts, directly derived from the classical proof of the Hahn-Banach theorem (see, for instance, [5], Theorem II.3.10).

Let $X$ be a real vector space and let $\sigma$ be a seminorm on $X$. Let also $Y \subset X$ be a vector subspace and let $\phi: Y \rightarrow \mathrm{R}$ be a linear functional such that $\phi(y) \leq \sigma(y), y \in Y$. For each $x \in X$ we set

$$
\begin{aligned}
& a(x)=\sup _{y \in Y}[-\phi(y)-\sigma(y+x)], \\
& b(x)=\inf _{y \in Y}[\sigma(y+x)-\phi(y)] .
\end{aligned}
$$

As in the proof of Theorem II.3.10 from [5], we have $a(x) \leq b(x)$ for all $x \in X$, and $a(y)=b(y)=\phi(y)$ if $y \in Y$. Moreover, if $\psi: X \rightarrow \mathrm{R}$ is any Hahn-Banach extension of $\phi$ (i.e., $\psi$ is linear and extends $\phi$, and $\psi(x) \leq \sigma(x)$, $x \in X)$, we have $\psi(x) \in L(x)$, where $L(x)=[a(x), b(x)]$, for all $x \in X$.

\section{Positive functionals on subspaces of fractions}

Let $\Omega$ be a compact Hausdorff space, let $\mathscr{Q} \subset C_{\mathrm{K}}(\Omega)$ be a multiplicative family, and let $C_{\mathrm{K}}(\Omega) / \mathcal{Q}$ be the algebra of fractions with numerators in $C_{\mathrm{K}}(\Omega)$, and with denominators in 2 .

Remark. We do not intend to define formally the concept of space of fractions. We shall work with various subspaces of the algebra of fractions $C_{\mathrm{K}}(\Omega) / \mathscr{Q}$, having suitable properties. For instance, if $\mathscr{Q}_{0} \subset \mathscr{Q}$ is nonempty, and if $\mathscr{S} \subset C_{\mathrm{K}}(\Omega)$ is a vector subspace, we may consider the subspace $\sum_{q \in \mathscr{L}_{0}} \mathscr{S} / q$ of $C_{\mathrm{K}}(\Omega) / \mathscr{Q}$, where $\mathscr{S} / q=\left\{f \in C_{\mathrm{K}}(\Omega) / q ; q f \in \mathscr{S}\right\}$. As $\mathscr{S} / q \subset C_{\mathrm{K}}(\Omega) / q$, 
$q \in \mathscr{Q}_{0}$, is a normed subspace, the space $\sum_{q \in \mathscr{Q}_{0}} \mathscr{S} / q$ is an inductive limit of normed spaces.

We use throughout the text the notation $q^{-1}$ to designate the element $1 / q$ for any $q \in \mathcal{Q}$.

Definition 3.1. Let $\mathscr{2}_{0} \subset \mathscr{Q}$ be nonempty. Let $\mathscr{F}=\sum_{q \in \mathscr{2}_{0}} C_{\mathrm{K}}(\Omega) / q$, and let $\psi: \mathscr{F} \rightarrow \mathrm{K}$ be linear. The functional $\psi$ is said to be positive if $\psi \mid\left(C_{\mathrm{K}}(\Omega) / q\right)^{+} \geq 0$ for all $q \in \mathscr{2}_{0}$.

THeOREM 3.2. Let $\mathscr{Q}_{0} \subset \mathscr{Q}$ be nonempty, let $\mathscr{F}=\sum_{q \in \mathscr{Q}_{0}} C_{\mathrm{K}}(\Omega) / q$, and let $\psi: \mathscr{F} \rightarrow \mathrm{K}$ be linear. The functional $\psi$ is positive if and only if

$$
\sup \left\{\left|\psi\left(h q^{-1}\right)\right| ; h \in C_{\mathrm{K}}(\Omega),\|h\|_{\infty} \leq 1\right\}=\psi\left(q^{-1}\right), \quad q \in \mathscr{Q}_{0} .
$$

If $\psi: \mathscr{F} \rightarrow \mathrm{C}$ is positive, there exists a unique positive Borel measure $\mu$ on $\Omega$ such that

$$
\psi(f)=\int_{\Omega} f d \mu, \quad f \in \mathscr{F} .
$$

In particular, the function $q^{-1}$ is $\mu$-integrable for all $q \in \mathscr{Q}_{0}$.

Proof. We use the (already mentioned) fact that a linear functional $\theta$ : $C_{\mathrm{K}}(\Omega) \rightarrow \mathrm{C}$ is positive if and only if it is continuous and $\|\theta\|=\theta(1)$

Set $\tilde{\psi}_{q}(h)=\psi\left(h q^{-1}\right), h \in C_{\mathrm{K}}(\Omega), q \in \mathscr{2}_{0}$.

Suppose $\psi$ positive. As $C_{\mathrm{K}}(\Omega) \subset \mathscr{F}$ and each positive function $h \in C_{\mathrm{K}}(\Omega)$ is also positive in $C_{\mathrm{K}}(\Omega) / q$, the functional $\tilde{\psi}_{q}$ is positive on $C_{\mathrm{K}}(\Omega)$. Hence

$$
\begin{aligned}
\left\|\tilde{\psi}_{q}\right\|=\sup \left\{\left|\psi\left(h q^{-1}\right)\right| ; h \in C_{\mathrm{K}}(\Omega),\right. & \left.\|h\|_{\infty} \leq 1\right\} \\
& =\tilde{\psi}_{q}(1)=\psi\left(q^{-1}\right), \quad q \in \mathscr{Q}_{0},
\end{aligned}
$$

which is the stated condition.

Conversely, the equality $\left\|\tilde{\psi}_{q}\right\|=\psi\left(q^{-1}\right)=\tilde{\psi}_{q}(1)$ shows that $\tilde{\psi}_{q}$ is positive on $C_{\mathrm{K}}(\Omega)$. Then there exists a positive (Borel) measure $\mu_{q}$ on $\Omega$ such that $\tilde{\psi}_{q}(h)=\int_{\Omega} h d \mu_{q}, h \in C_{\mathrm{K}}(\Omega)$, for all $q \in \mathscr{Q}_{0}$.

The relation $\tilde{\psi}_{q_{1}}\left(h q_{1}\right)=\psi(h)=\tilde{\psi}_{q_{2}}\left(h q_{2}\right)$ for all $q_{1}, q_{2} \in \mathscr{Q}_{0}$ and $h \in$ $C_{\mathrm{K}}(\Omega)$ implies the equality $q_{1} \mu_{q_{1}}=q_{2} \mu_{q_{2}}$. Therefore, there exists a positive measure $\mu$ such that $\mu=q \mu_{q}$ for all $q \in \mathscr{Q}_{0}$.

The equality $\mu=q \mu_{q}$ shows the set $\{\omega ; q(\omega)=0\}$ must be $\mu$-null. Consequently, $\mu_{q}=q^{-1} \mu$, and the function $q^{-1}$ is $\mu$-integrable for all $q \in \mathscr{Q}_{0}$.

If $f \in \mathscr{F}$ is arbitrary, then $f=\sum_{j \in J} h_{j} q_{j}^{-1}$, with $h_{j} \in C_{\mathrm{K}}(\Omega), q_{j} \in \mathscr{Q}_{0}$ for all $j \in J, J$ finite. We can write

$$
\psi(f)=\sum_{j \in J} \tilde{\psi}_{q_{j}}\left(h_{j}\right)=\sum_{j \in J} \int_{\Omega} h_{j} d \mu_{q_{j}}=\int_{\Omega} f d \mu .
$$


The measure $\mu$ being positive, the functional $\psi$ must be also positive.

The asserted integral representation for $\psi$ via the measure $\mu$ is now clear. Moreover, the measure $\mu$ is uniquely determined because of the equality $\psi(h)=\int_{\Omega} h d \mu, h \in C_{\mathrm{K}}(\Omega)$.

The measure $\mu$ on $\Omega$ with the property $\psi(f)=\int_{\Omega} f d \mu, f \in \mathscr{F}$, given by Theorem 3.2, is said to be a representing measure for the positive functional $\psi: \mathscr{F} \rightarrow \mathrm{C}$.

Remark 3.3. Let $\mathscr{F}=\sum_{q \in \mathscr{Q}_{0}} C_{\mathrm{K}}(\Omega) / q$ for a nonempty $\mathscr{Q}_{0} \subset \mathscr{Q}$, and let $\psi: \mathscr{F} \rightarrow \mathrm{C}$ be a positive functional on $\mathscr{F}$, with $\psi(1)>0$. If $\tilde{\psi}_{q}$ is defined as in the proof of Theorem 3.2, because $\tilde{\psi}_{q}(q)=\psi(1)>0$, and so $\tilde{\psi}_{q} \neq 0$, setting $\psi_{q}=\psi \mid C_{\mathrm{K}}(\Omega) / q$, it follows from the previous proof that

$$
\left\|\psi_{q}\right\|=\sup _{\|f\|_{\infty, q} \leq 1}|\psi(f)|=\sup _{\|h\|_{\infty} \leq 1}\left|\tilde{\psi}_{q}(h)\right|=\left\|\tilde{\psi}_{q}\right\|=\psi\left(q^{-1}\right)>0
$$

for all $q \in \mathscr{Q}_{0}$.

THEOREM 3.4. Let $\mathscr{Q}_{0} \subset \mathscr{Q}$ be nonempty and let $\mathscr{F}=\sum_{q \in \mathscr{L}_{0}} C_{\mathrm{K}}(\Omega) / q$. Let also $\mathscr{S}$ be a vector subspace of $\mathscr{F}$ such that $1 \in \mathscr{S}$ and $q^{-1} \in \mathscr{S}$ for all $q \in \mathscr{Q}_{0}$.

A linear functional $\phi: \mathscr{S} \rightarrow \mathrm{K}$ with $\phi(1)>0$ extends to a positive linear functional $\psi: \mathscr{F} \rightarrow \mathrm{K}$ if and only if $\phi\left(q^{-1}\right)>0, q \in \mathscr{Q}_{0}$, and $|\phi(g)| \leq \sigma_{V}(g), g \in \mathscr{S}$, where

$$
V=\operatorname{co}\left(\bigcup_{q \in \mathscr{Q}_{0}}\left\{f \in C_{\mathrm{K}}(\Omega) / q ;\|q f\|_{\infty} \leq \phi\left(q^{-1}\right)^{-1}\right\}\right) .
$$

Proof. Assume first that $\phi$ has a positive extension $\psi$. Then $\psi\left(q^{-1}\right)=$ $\phi\left(q^{-1}\right)>0$ for all $q \in \mathscr{Q}_{0}$, via Remark 3.3.

To prove the remaining condition, we note that we actually have $|\psi(f)| \leq$ $\sigma_{V}(f), f \in \mathscr{F}$. Indeed, $\psi$ being positive with $\psi(1)>0$, if $\psi_{q}$ is as in Remark 3.3, we have that $\left\|\psi_{q}\right\|=\psi\left(q^{-1}\right)>0$, and the assertion follows from Lemma 2.1(a). In particular, $|\phi(g)| \leq \sigma_{V}(g), g \in \mathscr{S}$, because $\psi$ is an extension of $\phi$.

Conversely, let $\phi: \mathscr{S} \rightarrow \mathrm{K}$ be a linear functional having the properties from the statement. The Hahn-Banach theorem implies the existence of an extension $\psi: \mathscr{F} \rightarrow \mathrm{K}$ with the property $|\psi(f)| \leq \sigma_{V}(f), f \in \mathscr{F}$. Then we have the estimate $\left\|\psi_{q}\right\| \leq \phi\left(q^{-1}\right)$, where $\psi_{q}=\psi \mid C_{K}(\Omega) / q$, via Lemma 2.1(b). The equality $\phi\left(q^{-1}\right)=\psi\left(q^{-1}\right)$ proves that $\left\|\psi_{q}\right\|=\psi\left(q^{-1}\right)$. An application of Theorem 3.2, via Remark 3.3, shows that $\psi$ must be positive. 
Remarks 3.5. (1) Let $q \in \mathscr{2}$ be fixed and let $\mathscr{S} \subset C_{\mathrm{K}}(\Omega) / q$ be such that $q^{-1} \in \mathscr{S}$. Let $\phi: \mathscr{S} \rightarrow \mathrm{K}$ be linear. If $\|\phi\|=\phi\left(q^{-1}\right)$, then $\phi$ has a positive extension $\psi: C_{\mathrm{K}}(\Omega) / q \rightarrow \mathrm{K}$, where $\|\phi\|$ is computed with respect to the norm of $C_{\mathrm{K}}(\Omega) / q$ restricted to $\mathscr{S}$. Indeed, if $\psi$ is a norm preserving extension of $\phi$ to $C_{\mathrm{K}}(\Omega) / q$, then $\|\psi\|=\psi\left(q^{-1}\right)$ and the assertion follows directly from Theorem 3.2 (also showing that the condition $\|\phi\|=\phi\left(q^{-1}\right)$ is necessary and that it insures the existence of a representing measure for $\phi$ ).

(2) As we have already noticed at the beginning of the second section, the gauge function of type $\sigma_{V}$ can be expressed in a slightly different manner. Let $\mathscr{Q}_{0} \subset \mathscr{Q}$ be nonempty, let $\mathscr{S}_{q}$ be a vector subspace of $C_{\mathrm{K}}(\Omega) / q$, thus $q \mathscr{S}_{q} \subset C_{\mathrm{K}}(\Omega)$, and let $\mathscr{F}=\sum_{q \in \mathscr{Q}_{0}} \mathscr{S}_{q}$. Let also $\delta=\left(\delta_{q}\right)_{q \in \mathscr{2}_{0}}$ be a family of positive numbers. Set

$$
\sigma_{\delta}(f)=\inf \left\{\sum_{k \in K} \delta_{q_{k}}\left\|h_{k}\right\|_{\infty} ; f=\sum_{k \in K} q_{k}^{-1} h_{k}, h_{k} \in q_{k} \mathscr{S}_{q_{k}}, k \in K, K \text { finite }\right\} .
$$

We have the equality $\sigma_{\delta}(f)=\sigma_{V}(f), f \in \mathscr{F}$, where $\sigma_{V}$ is the gauge function of the set

$$
V=\operatorname{co}\left(\bigcup_{q \in \mathscr{2}_{0}}\left\{f \in \mathscr{S}_{q} ;\|q f\|_{\infty} \leq \delta_{q}^{-1}\right\}\right) .
$$

With some supplementary conditions, one can obtain a version of Theorem 3.4 depending on simplified estimates (involving no gauge function). For this reason, the next theorem is more convenient for certain applications (see the next section).

Remark 3.6. In the family $\mathscr{Q}$ there is a natural partial ordering (reflexive and transitive but, in general, not antisymmetric), written as $q^{\prime} \mid q^{\prime \prime}$ for $q^{\prime}, q^{\prime \prime} \in$ 2 , meaning $q^{\prime}$ divides $q^{\prime \prime}$, that is, there exists a $q \in \mathscr{2}$ such that $q^{\prime \prime}=q^{\prime} q$. In particular, a subset $\mathscr{Q}_{0} \subset \mathscr{Q}$ is directed (resp. cofinal in $\mathscr{2}$ ) if for each pair $q^{\prime}, q^{\prime \prime} \in \mathscr{Q}_{0}$ there exists a $q \in \mathscr{Q}_{0}$ such that $q^{\prime} \mid q$ and $q^{\prime \prime} \mid q$ in $\mathscr{2}$ (resp. for every $q \in \mathscr{2}$ we can find a $q_{0} \in \mathscr{2}_{0}$ such that $q \mid q_{0}$ ). Because $\mathscr{2}$ is multiplicative, every cofinal subset of $\mathscr{Q}$ is directed.

Note also that if $q^{\prime}, q^{\prime \prime} \in \mathscr{Q}$ and $q^{\prime} \mid q^{\prime \prime}$, then $C_{\mathrm{K}}(\Omega) / q^{\prime} \subset C_{\mathrm{K}}(\Omega) / q^{\prime \prime}$, as one can easily see.

For every $q \in \mathscr{Q}$ we denote by $Z(q)$ the set $\{\omega \in \Omega ; q(\omega)=0\}$, that is, the zeros of $q$ in $\Omega$.

THeOREM 3.7. Let $\mathscr{Q}_{0}$ be a cofinal subset of 2 . Suppose $C_{\mathrm{K}}(\Omega)$ separable and that there exists a function $q_{0} \in \mathscr{2}_{0}$ with the property $Z\left(q_{0}\right)=\cup_{q \in \mathscr{Q}_{0}} Z(q)$. Let $\mathscr{F}=\sum_{q \in \mathscr{2}_{0}} \mathscr{F}_{q}$, where $\mathscr{F}_{q}$ is a vector subspace of $C_{\mathrm{K}}(\Omega) / q$ such that 
$1 \in \mathscr{F}_{q}, q^{-1} \in \mathscr{F}_{q}$, and $\mathscr{F}_{q} \subset \mathscr{F}_{q^{\prime}}$ for all $q, q^{\prime} \in \mathscr{Q}_{0}$, with $q \mid q^{\prime}$. Let also $\phi: \mathscr{F} \rightarrow \mathrm{K}$ be linear with $\phi(1)>0$, and set $\phi_{q}=\phi \mid \mathscr{F}_{q}$ for all $q \in \mathscr{Q}_{0}$.

The linear functional $\phi$ extends to a positive linear functional $\psi$ on $C_{\mathrm{K}}(\Omega) / 2$ such that $\left\|\psi_{q}\right\|=\left\|\phi_{q}\right\|$, where $\psi_{q}=\psi \mid C_{\mathrm{K}}(\Omega) / q$, if and only if $\left\|\phi_{q}\right\|=\phi\left(q^{-1}\right)>0$ for all $q \in \mathscr{Q}_{0}$.

PRoof. If $\phi: \mathscr{F} \rightarrow \mathrm{K}$ extends to a positive $\psi: C_{\mathrm{K}}(\Omega) / \mathscr{Q} \rightarrow \mathrm{K}$ such that $\left\|\psi_{q}\right\|=\left\|\phi_{q}\right\|$ for all $q \in \mathscr{2}_{0}$, then $\left\|\psi_{q}\right\|=\left\|\phi_{q}\right\|=\phi\left(q^{-1}\right)=\psi\left(q^{-1}\right)>$ 0 , by Theorem 3.2 and Remark 3.3. (Moreover, this is true with no special assumptions on the space $C_{\mathrm{K}}(\Omega)$, on $\left(\mathscr{F}_{q}\right)_{q \in \mathscr{Q}_{0}}$ or on $\mathscr{Q}_{0}$.)

Conversely, extend $\phi_{q}$ to a linear functional $\xi_{q}: C_{\mathrm{K}}(\Omega) / q \rightarrow \mathrm{K}$ such that $\left\|\xi_{q}\right\|=\left\|\phi_{q}\right\|=\phi\left(q^{-1}\right)$ for all $q \in \mathscr{Q}_{0}$. Set $\tilde{\xi}_{q}(h)=\xi_{q}\left(h q^{-1}\right), h \in C_{\mathrm{K}}(\Omega)$, $q \in \mathscr{2}_{0}$. We have $\left\|\tilde{\xi}_{q}\right\|=\left\|\xi_{q}\right\|=\phi\left(q^{-1}\right)=\tilde{\xi}_{q}(1)$ for all $q \in \mathscr{Q}_{0}$, as in Remark 3.3. Therefore, $\tilde{\xi}_{q}$ is positive on $C_{\mathrm{K}}(\Omega)$, and we can find a positive measure $v_{q}$ on $\Omega$ such that $\tilde{\xi}_{q}(h)=\int h d v_{q}$ for all $h \in C_{\mathrm{K}}(\Omega), q \in \mathscr{Q}_{0}$. This implies that the functional $\theta_{q}(h)=\tilde{\xi}_{q}(q h)$ is also positive on $C_{\mathrm{K}}(\Omega)$. Thus, we can find a measure $\mu_{q}$ on $\Omega$ such that $\theta_{q}(h)=\int h d \mu_{q}$ for all $h \in C_{\mathrm{K}}(\Omega)$, $q \in \mathscr{2}_{0}$. As in the proof of Theorem 3.2, the equality $\mu_{q}=q v_{q}$ shows that the set $\{\omega \in \Omega ; q(\omega)=0\}$ is $\mu_{q}$-null, and the function $q^{-1}$ is $\mu_{q}$-integrable for all $q \in \mathscr{2}_{0}$. Moreover,

$$
\phi(f)=\xi_{q}(f)=\tilde{\xi}_{q}(q f)=\int q f d v_{q}=\int f d \mu_{q}
$$

for all $f \in \mathscr{F}_{q}$ and $q \in \mathscr{Q}_{0}$. As a matter of fact, as we have $\phi_{q}=\phi_{q^{\prime}} \mid \mathscr{F}_{q}$, we infer that $\int f d \mu_{q}=\int f d \mu_{q^{\prime}}$ for all $f \in \mathscr{F}_{q} \subset \mathscr{F}_{q^{\prime}}$, when $q \mid q^{\prime}$, via the hypothesis. Note also that $\left\|\mu_{q}\right\|=\int d \mu_{q}=\phi(1)$ for all $q \in \mathscr{Q}_{0}$, by the positivity of $\mu_{q}$. Therefore, the family $\left(\mu_{q}\right)_{q \in \mathscr{2}_{0}}$ is bounded in the dual $M_{\mathrm{K}}(\Omega)$ of $C_{\mathrm{K}}(\Omega)$, which is constantly identified with the space of finite Borel measures on $\Omega$.

Let $M_{q}$ be the weak*-closure of the set $\left\{\mu_{q^{\prime}} ; q^{\prime} \in \mathscr{Q}_{0}, q \mid q^{\prime}\right\}$ in $M_{\mathrm{K}}(\Omega)$, for all $q \in \mathscr{Q}_{0}, q_{0} \mid q$, with $q_{0}$ as in the statement. Note also that, for all finite families $\left\{q_{1}, \ldots, q_{m}\right\}$ of elements of $\mathscr{Q}_{0}$ divided by $q_{0}$, we can find a $q \in \mathscr{Q}_{0}$ divided by $q_{1}, \ldots, q_{m}$, because the set $\mathscr{Q}_{0}$ is directed, and so $M_{q} \subset$ $M_{q_{1}} \cap \cdots \cap M_{q_{m}}$. This shows that $\cap_{q \in \mathscr{Q}_{0}, q_{0} \mid q} M_{q}$ is nonempty in $M_{\mathrm{K}}(\Omega)$, via the weak*-compactness of its bounded sets.

Fix an element $\mu \in \cap_{q \in \mathscr{Q}_{0}, q_{0} \mid q} M_{q}$, regarded as a measure on $\Omega$. Because the space $C_{\mathrm{K}}(\Omega)$ is separable, the weak*-topology of the bounded sets in $M_{\mathrm{K}}(\Omega)$ is metrizable (see [8], Theorem 2.6.23). Therefore, there exists a sequence $\left(\mu_{q_{k}}\right)_{k \geq 1}$ in $M_{q_{0}}$ which is weakly*-convergent to $\mu \in M_{q_{0}}$. As $\int h d \mu=$ $\lim _{k \rightarrow \infty} \int h d \mu_{q_{k}}, h \in C_{\mathrm{K}}(\Omega)$, we clearly have $\mu$ positive and $\int d \mu=\phi(1)$. 
Put $N=Z\left(q_{0}\right)$. Note that the set $N$ is $\mu_{q}$-null for each $q \in \mathscr{Q}_{0}$ with $q_{0} \mid q$, as a consequence of a previous remark and the hypothesis on $q_{0}$. We want to show that the functions from $\sum_{q \in \mathscr{2}_{0}} C_{\mathrm{K}}(\Omega) / q$, restricted to $\Omega \backslash N$, are all $\mu$-integrable. It suffices to show that each function $q^{-1}$, restricted to $\Omega \backslash N$, with $q \in \mathscr{2}_{0}$, is $\mu$-integrable. We refine some ideas from [14] (see [14], Proposition 1).

Fix a function $q \in \mathscr{Q}_{0}$. Note that if $q^{\prime} \in \mathscr{2}_{0}$ is divided by $q$ and $q^{\prime-1}$ is $\mu$ integrable, then $q^{-1}$ is $\mu$-integrable. Therefore, since $\mathscr{Q}_{0}$ is directed, we may assume, with no loss of generality, that $q_{0} \mid q$.

Let $\left(\mu_{q_{k}}\right)_{k \geq 1}$ be a sequence in $M_{q}$ which is weakly*-convergent to $\mu$. As the set $\Omega$ is compact and metrizable (see [8], Ex. 2.50), we can find a sequence of compact subsets $\left(\Omega_{m}\right)_{m \geq 1}$ such that $\Omega_{m}$ is a subset of the interior of $\Omega_{m+1}$ for all $m \geq 1$, and $\cup_{m \geq 1} \Omega_{m}=\Omega \backslash N$. We also choose a sequence of functions $\left(g_{m}\right)_{m \geq 1}$ in $C_{\mathrm{K}}(\Omega)$, having values in the interval $[0,1]$, with $g_{m} \mid \Omega_{m}=1$ and the support of $g_{m}$ included in the interior of $\Omega_{m+1}$ for all $m \geq 1$. By Fatou's lemma, we have:

$$
\begin{aligned}
& \int_{\Omega \backslash N} q^{-1} d \mu=\int \lim _{m \rightarrow \infty} g_{m} q^{-1} d \mu \leq \liminf _{m \rightarrow \infty} \int g_{m} q^{-1} d \mu \\
= & \liminf _{m \rightarrow \infty} \lim _{k \rightarrow \infty} \int g_{m} q^{-1} d \mu_{q_{k}} \leq \lim _{k \rightarrow \infty} \int q^{-1} d \mu_{q_{k}}=\phi\left(q^{-1}\right),
\end{aligned}
$$

because $q^{-1} \in \mathscr{F}_{q_{k}}$ for all $k \geq 1$, in virtue of the hypothesis on the family $\left(\mathscr{F}_{q}\right)_{q \in \mathscr{2}_{0}}$.

Consequently, all functions in $\sum_{q \in \mathscr{Q}_{0}} C_{\mathrm{K}}(\Omega) / q$, restricted to $\Omega \backslash N$, are $\mu$-integrable. As a matter of fact, we have the equality $\sum_{q \in \mathscr{2}_{0}} C_{\mathrm{K}}(\Omega) / q=$ $C_{\mathrm{K}}(\Omega) / \mathscr{Q}$ because the family $\mathscr{Q}_{0}$ is cofinal in $\mathscr{Q}$ (see Remark 3.6).

We now prove the equality $\phi(f)=\int_{\Omega \backslash N} f d \mu, f \in \mathscr{F}$. It suffices to take an $f=h q^{-1} \in \mathscr{F}_{q}$ with $q \in \mathscr{2}_{0}$, and so $h \in C_{\mathrm{K}}(\Omega)$. We can find a function $q^{\prime} \in \mathscr{2}_{0}$ such that $q q_{0} \mid q^{\prime}$, because $\mathscr{2}_{0}$ is cofinal in $\mathscr{2}$. Then, with a sequence $\left(\mu_{q_{k}}\right)_{k \geq 1}$ weakly*-convergent to $\mu$ in $M_{q^{\prime}}$ and $\left(g_{m}\right)_{m \geq 0}$ as above,

$$
\begin{gathered}
\int_{\Omega \backslash N} f d \mu=\lim _{m \rightarrow \infty} \int g_{m} h q_{0}\left(q q_{0}\right)^{-1} d \mu=\lim _{m \rightarrow \infty} \lim _{k \rightarrow \infty} \int g_{m} h q_{0}\left(q q_{0}\right)^{-1} d \mu_{q_{k}} \\
=\lim _{k \rightarrow \infty} \lim _{m \rightarrow \infty} \int g_{m} h q_{0}\left(q q_{0}\right)^{-1} d \mu_{q_{k}}=\lim _{k \rightarrow \infty} \int h q_{0}\left(q q_{0}\right)^{-1} d \mu_{q_{k}} \\
=\int f d \mu_{q}=\phi_{q}(f)=\phi(f),
\end{gathered}
$$

as noticed before, since $q \mid q_{k}$ for all $k$. The two previous limits are interchangeable because the function $h q_{0}$ is null on $N$, and so the sequence $\left(g_{m} h q_{0}\right)_{m \geq 1}$ 
is uniformly convergent to $h q_{0}$. In addition, the inclusion $\left(q q_{0}\right)^{-1} \in \mathscr{F}_{q_{k}}$ implies $\int\left(q q_{0}\right)^{-1} d \mu_{q_{k}}=\phi\left(\left(q q_{0}\right)^{-1}\right), k \geq 1$, and thus the sequence of measures $\left(\left(q q_{0}\right)^{-1} \mu_{q_{k}}\right)_{k \geq 1}$ is norm bounded.

Similarly, we have:

$$
\begin{gathered}
\int_{N} d \mu=\lim _{m \rightarrow \infty} \int\left(1-g_{m}\right) q_{0} q_{0}^{-1} d \mu=\lim _{m \rightarrow \infty} \lim _{k \rightarrow \infty} \int\left(1-g_{m}\right) q_{0} q_{0}^{-1} d \mu_{q_{k}} \\
=\lim _{k \rightarrow \infty} \lim _{m \rightarrow \infty} \int\left(1-g_{m}\right) q_{0} q_{0}^{-1} d \mu_{q_{k}}=0,
\end{gathered}
$$

for a sequence $\left(\mu_{q_{k}}\right)_{k \geq 1}$ in $M_{q_{0}}$ which is weakly*-convergent to $\mu$, because of the uniform convergence of the sequence $\left(\left(1-g_{m}\right) q_{0}\right)_{m \geq 1}$ to 0 and the boundedness of the sequence of measures $\left(q_{0}^{-1} \mu_{q_{k}}\right)_{k \geq 1}$, showing that the set $N$ is $\mu$-null.

If we denote by $\psi$ the linear functional $f \rightarrow \int_{\Omega} f d \mu, f \in C_{\mathrm{K}}(\Omega) / \mathcal{Q}$, then $\psi$ is a positive extension of $\phi$. The equalities $\left\|\psi_{q}\right\|=\psi\left(q^{-1}\right)=\phi\left(q^{-1}\right)=$ $\left\|\phi_{q}\right\|, q \in \mathscr{Q}_{0}$, follow from Theorem 3.2.

Remarks 3.8. (1) The previous theorem gives an extension $\psi$ of $\phi$ whose representing measure $\mu$ has the property $\mu(N)=0$, where $N=\cup_{q \in \mathscr{Q}} Z(q)=$ $Z\left(q_{0}\right)$.

(2) To avoid some technical complications, it is sometimes useful to replace the partial ordering "|" in the family $\mathscr{Q}$ (see Remark 3.6) by a stronger one " $\uparrow$ " in the sense that $q^{\prime} \uparrow q^{\prime \prime}$ implies $q^{\prime} \mid q^{\prime \prime}$. The proof of Theorem 3.7 still holds, with minor modifications, by assuming the set $\mathscr{Q}$ endowed with a partial ordering "个" stronger that "l”, and the set $\mathscr{Q}_{0}$ cofinal in $\mathscr{Q}$ with respect to this stronger partial ordering.

\section{Application to moment problems}

In this section, we apply some of the preceding results to get information related to power moment problems in unbounded sets. We restrict ourselves to real moment problems, the complex case versions being similar.

Let us denote by $Z_{+}^{n}$ the set of all multi-indices $\alpha=\left(\alpha_{1}, \ldots, \alpha_{n}\right)$, i.e., $\alpha_{j} \in \mathbf{Z}_{+}$for all $j=1, \ldots, n$, with $\mathbf{Z}_{+}$the nonnegative part of the ring of integers $\mathbf{Z}$.

Let $\left(\mathbf{R}_{\infty}\right)^{n}=(\mathbf{R} \cup\{\infty\})^{n}$, i.e., the Cartesian product of $n$ copies of the one point compactification $R_{\infty}=R \cup\{\infty\}$ of the real line $R$. We consider the family $\mathscr{Q}_{n}$ consisting of all rational functions of the form $q_{\alpha}(t)=(1+$ $\left.t_{1}^{2}\right)^{-\alpha_{1}}, \ldots,\left(1+t_{n}^{2}\right)^{-\alpha_{n}}, t=\left(t_{1}, \ldots, t_{n}\right) \in \mathrm{R}^{n}$, where $\alpha=\left(\alpha_{1}, \ldots, \alpha_{n}\right) \in \mathrm{Z}_{+}^{n}$ is arbitrary. The function $q_{\alpha}$ can be continuously extended to $\left(\mathbf{R}_{\infty}\right)^{n} \backslash \mathbf{R}^{n}$ for all $\alpha \in \mathrm{Z}_{+}^{n}$. Moreover, the set $\mathscr{Q}_{n}$ becomes a multiplicative family in $C_{\mathbf{R}}\left(\left(\mathrm{R}_{\infty}\right)^{n}\right)$. Set also $p_{\alpha}(t)=q_{\alpha}(t)^{-1}, t \in \mathbf{R}^{n}, \alpha \in \mathbf{Z}_{+}^{n}$. 
Let $\mathscr{P}_{n}$ be the algebra of all polynomial functions on $\mathrm{R}^{n}$, with real coefficients. Let $\mathscr{P}_{n, \alpha}$ be the vector space generated by the monomials $t^{\beta}=$ $t_{1}^{\beta_{1}} \cdots t_{n}^{\beta_{n}}$, with $\beta_{j} \leq 2 \alpha_{j}, j=1, \ldots, n, \alpha \in \mathbf{Z}_{+}^{n}$. It is clear that $p / p_{\alpha}$ can be continuously extended to $\left(\mathbf{R}_{\infty}\right)^{n} \backslash \mathbf{R}^{n}$ for every $p \in \mathscr{P}_{n, \alpha}$, and so it can be regarded as an element of $C_{\mathrm{R}}\left(\left(\mathrm{R}_{\infty}\right)^{n}\right)$. Therefore, $\mathscr{P}_{n, \alpha}$ is a subspace of $C_{\mathrm{R}}\left(\left(\mathrm{R}_{\infty}\right)^{n}\right) / q_{\alpha}=p_{\alpha} C_{\mathrm{R}}\left(\left(\mathrm{R}_{\infty}\right)^{n}\right)$ for all $\alpha \in \mathrm{Z}_{+}^{n}$.

Let $\gamma=\left(\gamma_{\alpha}\right)_{\alpha \in \mathrm{Z}_{+}^{n}}$ be an $n$-sequence of real numbers and let $L_{\gamma}: \mathscr{P}_{n} \rightarrow \mathrm{C}$ be the associated linear functional given by $L_{\gamma}\left(t^{\alpha}\right)=\gamma_{\alpha}, \alpha \in Z_{+}^{n}$, extended by linearity. Let also $K \subset \mathrm{R}^{n}$ be a closed subset. Recall that the $n$-sequence $\gamma=\left(\gamma_{\alpha}\right)_{\alpha \in Z_{+}^{n}}$ of real numbers is said to be a $K$-moment sequence if there exists a positive measure $\mu$ on $K$ such that $t^{\alpha} \in L^{1}(\mu)$ and $\gamma_{\alpha}=\int_{K} t^{\alpha} d \mu(t)$, $\alpha \in Z_{+}^{n}$. The measure $\mu$ is said to be a representing measure for $\gamma$ (see, for instance, [2]). To avoid the trivial solution, one usually requires that $\gamma_{0}>0$.

First of all, let us state a characterization of the moment sequences on $\mathrm{R}^{n}$, i.e., the $\mathrm{R}^{n}$-moment sequences.

THEOREM 4.1. An n-sequence $\gamma=\left(\gamma_{\alpha}\right)_{\alpha \in \mathrm{Z}_{+}^{n}}\left(\gamma_{0}>0\right)$ of real numbers is a moment sequence on $\mathrm{R}^{n}$ if and only if the associated linear functional $L_{\gamma}: \mathscr{P}_{n} \rightarrow \mathrm{R}$ has the properties $L_{\gamma}\left(p_{\alpha}\right)>0$ and

$$
\left|L_{\gamma}(p)\right| \leq L_{\gamma}\left(p_{\alpha}\right) \sup _{t \in \mathrm{R}^{n}}\left|q_{\alpha}(t) p(t)\right|, \quad p \in \mathscr{P}_{n, \alpha}, \alpha \in \mathrm{Z}_{+}^{n} .
$$

Theorem 4.1 follows directly from Theorem 4.2, which characterizes the $K$-moment sequences, in general.

Remarks. (1) Defining the partial ordering $\xi \prec \eta$ for two multi-indices $\xi=\left(\xi_{1}, \ldots, \xi_{n}\right), \eta=\left(\eta_{1}, \ldots, \eta_{n}\right)$, meaning that $\xi_{j} \leq \eta_{j}, j=1, \ldots, n$, we can rephrase the conditions from Theorem 4.1 as follows: $\gamma=\left(\gamma_{\alpha}\right)_{\alpha \in Z_{+}^{n}}$ is a moment sequence on $\mathbf{R}^{n}$ if and only if $L_{\gamma}\left(p_{\alpha}\right)>0$ and

$$
\left|\sum_{\beta<2 \alpha} a_{\beta} \gamma_{\beta}\right| \leq L_{\gamma}\left(p_{\alpha}\right) \sup _{t}\left|\sum_{\beta<2 \alpha} a_{\beta} t^{\beta} q_{\alpha}(t)\right|
$$

for all finite sequences of real numbers $\left(a_{\beta}\right)_{\beta<2 \alpha}$ and all $\alpha \in \mathrm{Z}_{+}^{n}$, via the explicit structure of the space $\mathscr{P}_{n, \alpha}$.

(2) For a fixed $\alpha \in Z_{+}^{n}$, we may consider a finite sequence $\gamma=\left(\gamma_{\beta}\right)_{\beta<2 \alpha}$ $\left(\gamma_{0}>0\right)$. Remark 3.5(1) leads directly to a characterization of all such sequences $\gamma$ having a representing measure, i.e., a positive measure on $\mathrm{R}^{n}$ (or on a closed subset of it) such that $\gamma_{\beta}=\int t^{\beta} d \mu(t)$ for all $\beta \prec 2 \alpha$. A necessary and sufficient condition is that

$$
\sup \left\{\left|L_{\gamma}(p)\right| ; \sup _{t \in \mathrm{R}^{n}}\left|q_{\alpha}(t) p(t)\right| \leq 1\right\}=L_{\gamma}\left(p_{\alpha}\right)>0,
$$


which is equivalent to the condition in (1), for a fixed $\alpha$.

The moment problem for finite sequences, i.e., the truncated moment problem, is extensively developed in [3] and [4].

Fix a set closed $K \subset \mathbf{R}^{n}$. If $K$ is bounded, an $n$-sequence $\gamma=\left(\gamma_{\alpha}\right)_{\alpha \in \mathrm{Z}_{+}^{n}}$ is a $K$-moment sequence if and only if $\left|L_{\gamma}(p)\right| \leq \gamma_{0} \sup _{t \in K}|p(t)|, p \in \mathscr{P}_{n}$, which is equivalent to the existence of a positive extension of $L_{\gamma}$ to $C_{\mathrm{R}}(K)$. As we are primarily interested to apply this point of view to the unbounded case, we will assume, in general, that $K$ is unbounded.

Remark. Let $K \subset \mathrm{R}^{n}$ be closed and unbounded, and let $\Omega$ be the closure of $K$ in the compact space $\left(\mathrm{R}_{\infty}\right)^{n}$. Let also $\mathscr{Q}_{n}(\Omega)$ be the set of functions from $\mathscr{Q}_{n}$, (extended to $\left(\mathrm{R}_{\infty}\right)^{n}$ and) restricted to $\Omega$. Note that the map $\mathscr{Q}_{n} \ni q \rightarrow$ $q \mid \Omega \in \mathscr{Q}_{n}(\Omega)$ is not necessarily injective.

If $q^{\prime}, q^{\prime \prime} \in \mathscr{Q}_{n}(\Omega)$, we write $q^{\prime} \uparrow q^{\prime \prime}$ if for any $\alpha \in \mathrm{Z}_{+}^{n}$ with the property $q^{\prime}=q_{\alpha} \mid \Omega$, we can find a $\beta \in Z_{+}^{n}$ such that $q^{\prime \prime}=q_{\beta} \mid \Omega$ and $\beta-\alpha \in Z_{+}^{n}$. This can be easily seen to be a partial ordering stronger than the division (see Remark 3.6 and Remark 3.8(2)).

For every $q \in \mathscr{Q}_{n}(\Omega)$, let $\mathscr{P}_{n, q}$ be the space of polynomial functions $p \mid K$, $p \in \mathscr{P}_{n}$, such that the function $(p q) \mid K$ has a continuous extension to $\Omega$.

Let $\mathscr{P}_{n, K}$ be the space $\left\{p \mid K ; p \in \mathscr{P}_{n}\right\}$. Then we have $\mathscr{P}_{n, K}=\sum_{q \in \mathscr{Q}_{n}(\Omega)} \mathscr{P}_{n, q}$, and $\mathscr{P}_{n, K}$ can be regarded as a subspace of the algebra of fractions $C_{\mathrm{R}}(\Omega) / \mathscr{Q}_{n}(\Omega)$, where each space $\mathscr{P}_{n, q}$ is a subspace of $C_{\mathrm{R}}(\Omega) / q, q \in \mathscr{Q}_{n}(\Omega)$.

When the map $L_{\gamma}$ has the property $L_{\gamma}(p)=0$ for every $p \in \mathscr{P}_{n}$ with $p \mid K=0$, then $L_{\gamma}$ induces a linear map on $\mathscr{P}_{n, K}$, which will be also denoted by $L_{\gamma}$.

With these remarks and notation, we have the following.

THEOREM 4.2. An n-sequence $\gamma=\left(\gamma_{\alpha}\right)_{\alpha \in Z_{+}^{n}}\left(\gamma_{0}>0\right)$ of real numbers is a $K$-moment sequence if and only if the associated linear functional $L_{\gamma}$ : $\mathscr{P}_{n} \rightarrow \mathrm{R}$ has the properties $L_{\gamma}(p)=0$ for every $p \in \mathscr{P}_{n}$ with $p \mid K=0$, $L_{\gamma}\left(q^{-1}\right)>0$ and

$$
\left|L_{\gamma}(p)\right| \leq L_{\gamma}\left(q^{-1}\right) \sup _{t \in K}|q(t) p(t)|, \quad p \in \mathscr{P}_{n, q}, q \in \mathscr{Q}_{n}(\Omega) .
$$

Proof. If $\gamma$ is a $K$-moment sequence, and so $L_{\gamma}(p)=\int_{K} d d \mu, p \in \mathscr{P}_{n}$, for a certain positive measure $\mu$ on $K$, as $q^{-1} \geq 1$ on $K$, it follows $L_{\gamma}\left(q^{-1}\right) \geq$ $\int_{K} d \mu=\gamma_{0}>0$ for all $q \in \mathscr{Q}_{n}(\Omega)$. In addition,

$$
\left|L_{\gamma}(p)\right| \leq \int_{K}|p| d \mu \leq L_{\gamma}\left(q^{-1}\right) \sup _{t \in K}|q(t) p(t)|,
$$


for all $p \in \mathscr{P}_{n, q}$ and $q \in \mathscr{Q}_{n}(\Omega)$, which is the desired estimate.

To prove that the stated conditions imply the existence of a representing measure for the sequence $\gamma$, we shall apply Theorem 3.7 to the space $\mathscr{P}_{n, K} \subset$ $C_{\mathrm{R}}(\Omega) / \mathscr{Q}_{n}(\Omega)$ and the map $L_{\gamma}: \mathscr{P}_{n, K} \rightarrow \mathrm{R}$. Note first that the space $C_{\mathrm{R}}(\Omega)$ is separable. Indeed, the family of functions $\left(1+t_{1}^{2}\right)^{-1}, t_{1}\left(1+t_{1}^{2}\right)^{-1}, \ldots,(1+$ $\left.t_{n}^{2}\right)^{-1}, t_{n}\left(1+t_{n}^{2}\right)^{-1}$, extended to $\left(\mathbf{R}_{\infty}\right)^{n}$, separates the points of $\left(\mathbf{R}_{\infty}\right)^{n}$, and therefore it separates the points of $\Omega$. Then the Weierstrass-Stone theorem implies the density of the unital algebra generated by this family in $C_{\mathrm{R}}(\Omega)$. Note also that the function $q_{(1, \ldots, 1)} \mid \Omega$ is null on the set $\Omega \backslash K$, and this set contains the zeros of any function from $\mathscr{Q}_{n}(\Omega)$. Notice also that $1, q^{-1} \in \mathscr{P}_{n, q} \subset C_{\mathrm{R}}(\Omega) / q$ for all $q \in \mathscr{Q}_{n}(\Omega)$. Moreover, we have $\mathscr{P}_{n, q^{\prime}} \subset \mathscr{P}_{n, q^{\prime \prime}}$, whenever $q^{\prime} \uparrow q^{\prime \prime}$, as one can easily see.

If $\phi_{q}=L_{\gamma} \mid \mathscr{P}_{n, q}$, considering $\|f\|_{\infty, q}=\sup _{t \in K}|q(t) f(t)|, f \in C_{\mathrm{R}}(\Omega) / q$, which is precisely the norm on $C_{\mathrm{R}}(\Omega) / q$, the conditions from the statement imply the estimates $\left\|\phi_{q}\right\| \leq L_{\gamma}\left(q^{-1}\right)$. As $q^{-1} \in \mathscr{P}_{n, q}$ is a norm one element, the conditions from the statement imply the conditions $\left\|\phi_{q}\right\|=L_{\gamma}\left(q^{-1}\right)>0$, $q \in \mathscr{Q}_{n}(\Omega)$, which, in turn, imply the existence of a positive extension $\Psi$ of $L_{\gamma}$ to $C_{\mathrm{R}}(\Omega) / \mathscr{Q}_{n}(\Omega)$, by Theorem 3.7. The proof of Theorem 3.7 shows, in fact, the existence of a representing measure of $\Psi$, and therefore of $L_{\gamma}$, whose support can be chosen to lie $K$, by Remark 3.8(1).

Remarks 4.3. (1) If the set $K$ in Theorem 4.2 has a nonempty interior, and so the map $\mathscr{Q}_{n} \ni q \rightarrow q \mid \Omega \in \mathscr{Q}_{n}(\Omega)$ is bijective, the existence of a representing measure for $\gamma$ can be characterized by the conditions $L_{\gamma}\left(p_{\alpha}\right)>0$ and $\left|L_{\gamma}(p)\right| \leq L_{\gamma}\left(p_{\alpha}\right) \sup _{t \in K}\left|q_{\alpha}(t) p(t)\right|, p \in \mathscr{P}_{n, \alpha}, \alpha \in Z_{+}^{n}$. The proof is similar to that of Theorem 4.2. One uses the spaces $\mathscr{P}_{n, \alpha}(K)=\{p \mid K ; p \in$ $\left.\mathscr{P}_{n, \alpha}\right\}$, noticing that $\mathscr{P}_{n, K}=\sum_{\alpha \in Z_{+}^{n}} \mathscr{P}_{n, \alpha}(K)$. The partial ordering of $\mathscr{Q}_{n}(\Omega)$ is induced by the partial ordering of $\mathscr{Q}_{n}$ (which, in turn, is induced by that of $Z_{+}^{n}$, introduced after Theorem 4.1). We also note that the conditions above imply the property $L_{\gamma}(p)=0$ if $p \mid K=0$. Other details are left to the reader. Theorem 4.1 is a consequence of this form of Theorem 4.2.

(2) The conditions above, as well as those in Theorem 4.1, may be replaced by similar conditions, in which the multi-index $\alpha$ runs only an a cofinal family in $Z_{+}^{n}$, which suffices to apply Theorem 3.7.

We can obtain the classical result of Haviland [7] as a consequence of Theorem 4.2.

Corollary 4.4. An n-sequence $\gamma=\left(\gamma_{\alpha}\right)_{\alpha \in \mathrm{Z}_{+}}\left(\gamma_{0}>0\right)$ of real numbers is a $K$-moment sequence if and only if the linear functional $L_{\gamma}: \mathscr{P}_{n} \rightarrow \mathrm{R}$ has the property $p \mid K \geq 0$ implies $L_{\gamma}(p) \geq 0$ for all $p \in \mathscr{P}_{n}$. 
Proof. The case $K$ compact being well known, we may assume $K$ unbounded. We use the notation related to Theorem 4.2.

Assume first that for all $p \in \mathscr{P}_{n}$, with $p \mid K \geq 0$, we have $L_{\gamma}(p) \geq 0$. This clearly implies $L_{\gamma}(p)=0$ for all $p \in \mathscr{P}_{n}$ with $p \mid K=0$, and shows that the map $L_{\gamma}: \mathscr{P}_{n, K} \rightarrow \mathrm{R}$ is well defined.

Now, let $q \in \mathscr{Q}_{n}(\Omega)$, let $\alpha \in \mathrm{Z}_{+}^{n}$ be such that $q=q_{\alpha} \mid K$, and let $p \in \mathscr{P}_{n, q}$. We have the estimate

$$
\pm p(t) \leq p_{\alpha}(t) \sup _{s \in K}|q(s) p(s)|, \quad t \in K .
$$

This leads to the relation $\left|L_{\gamma}(p)\right| \leq L_{\gamma}\left(p_{\alpha}\right) \sup _{s \in K}|q(s) p(s)|$. Since $p_{\alpha} \geq 1$, and so $L_{\gamma}\left(q^{-1}\right)=L_{\gamma}\left(p_{\alpha}\right) \geq L_{\gamma}(1)=\gamma_{0}>0$ for all $\alpha \in Z_{+}^{n}$, we may apply Theorem 4.2, showing that $\gamma$ is a $K$-moment sequence.

Conversely, if $\gamma$ is a $K$-moment sequence, it is clear that $p \mid K \geq 0$ implies $L_{\gamma}(p) \geq 0$ for all $p \in \mathscr{P}_{n}$, via the existence of a representing measure for $\gamma$ on $K$.

Remark. Let $K \subset \mathrm{R}^{n}$ be closed and unbounded, let $\Omega$ be the closure of $K$ in the space $\left(\mathbf{R}_{\infty}\right)^{n}$, and let $\gamma=\left(\gamma_{\alpha}\right)_{\alpha \in \mathbf{Z}_{+}^{n}}$ be an $n$-sequence of real numbers such that $L_{\gamma}(p)=0$ whenever $p \mid K=0$. Then, as we have already seen, the map $L_{\gamma}$ induces a linear map, also denoted by $L_{\gamma}$, on the space $\mathscr{P}_{n, K}$. In particular, for each $q \in \mathscr{Q}_{n}(\Omega)$, the symbol $L_{\gamma}\left(q^{-1}\right)$ is well defined.

For every pair $\xi, \eta \in \mathrm{Z}_{+}^{n}$, we set $r_{\xi, \eta}(t)=t^{\xi} q_{\eta}(t), t \in \mathrm{R}^{n}$. Let also $\mathscr{G}_{0}=\left\{r_{\xi, \eta} ; \xi, \eta \in \mathrm{Z}_{+}^{n}, \xi \prec 2 \eta\right\}$. We clearly have $\mathscr{G}_{0} \subset C_{\mathrm{R}}\left(\left(\mathrm{R}_{\infty}\right)^{n}\right)$.

As in Remark 2.4, for every $r \in \mathscr{G}_{0}$ we set

$$
\begin{aligned}
& a(r, q)=\sup _{p \in \mathscr{P}_{n, q}}\left[-L_{\gamma}(p)-L_{\gamma}\left(q^{-1}\right) \sup _{t \in K}|q(t)(p(t)+r(t))|\right], \\
& b(r, q)=\inf _{p \in \mathscr{P}_{n, q}}\left[L_{\gamma}\left(q^{-1}\right) \sup _{t \in K}|q(t)(p(t)+r(t))|-L_{\gamma}(p)\right],
\end{aligned}
$$

and we have $a(r, q) \leq b(r, q)$ for all $q \in \mathscr{Q}_{n}(\Omega)$. Thus, we can construct the interval $J_{q}(r)=[a(r, q), b(r, q)]$. Set $J(r)=\cap_{q \in \mathscr{Q}_{n}(\Omega)} J_{q}(r)$.

With the notation above, we have the following.

THEOREM 4.5. Let $\gamma=\left(\gamma_{\alpha}\right)_{\alpha \in \mathrm{Z}_{+}^{n}}\left(\gamma_{0}>0\right)$ be a $K$-moment sequence. The representing measure of $\gamma$ is uniquely determined if and only if $J(r)$ is singleton for all $r \in \mathscr{G}_{0}$, that is, if and only if

$$
\sup _{q \in \mathscr{Q}_{n}(\Omega)} a(r, q)=\inf _{q \in \mathscr{Q}_{n}(\Omega)} b(r, q),
$$

for all $r \in \mathscr{G}_{0}$. 
PRoOF. We remark that any representing measure $\mu$ of the sequence $\gamma$, whose support lies in $K$, gives rise to an extension $\psi$ of $L_{\gamma}$ to $C_{\mathrm{R}}(\Omega) / \mathscr{Q}_{n}(\Omega)$, in the following way. If $f \in C_{\mathrm{R}}(\Omega) / q$, then $f$ is $\mu$-integrable since $\int_{K}|f| d \mu \leq$ $L_{\gamma}\left(q^{-1}\right) \sup _{t \in K}|q(t) f(t)|$. We set $\psi(f)=\int_{K} f d \mu$ for all $f \in C_{\mathrm{R}}(\Omega) / \mathscr{Q}_{n}(\Omega)$. In fact, the map $\psi \mid C_{\mathrm{R}}(\Omega) / q$ is a Hahn-Banach extension of the map $L_{\gamma} \mid \mathscr{P}_{n, q}$ for all $q \in \mathscr{Q}_{n}(\Omega)$ (see Remark 2.4). In particular, $\psi(r) \in J(r)=\cap_{q \in \mathscr{Z}_{n}(\Omega)} J_{q}(r)$ for all $r \in \mathscr{G}_{0}$.

Let $J(r)$ be singleton for all $r \in \mathscr{G}_{0}$, and let $\mu_{1}, \mu_{2}$ be two representing measures for the sequence $\gamma$. Then for all $r \in \mathscr{G}_{0}$ we have the equality $\int_{K} r d \mu_{1}=\int_{K} r d \mu_{2}$, because $\int_{K} r d \mu_{1}, \int_{K} r d \mu_{2} \in J(r)$. As the set $\mathscr{G}_{0}$ generates a dense subspace of $C_{\mathrm{R}}(\Omega)$, we must have $\mu_{1}=\mu_{2}$.

Conversely, suppose that $\gamma$ has a uniquely determined representing measure whose support lies in $K$. In particular, the map $L_{\gamma}$ satisfies the conditions in Theorem 4.2. In particular, there is a map, say $\phi$, induced by $L_{\gamma}$ in $\mathscr{P}_{n, K}$. Note also that $\left\|\phi_{q}\right\|=\phi\left(q^{-1}\right)>0$ for all $q \in \mathscr{Q}_{n}(\Omega)$, as in the proof of Theorem 4.2, where $\phi_{q}=\phi \mid \mathscr{P}_{n, q}$.

Assume the existence of an $r_{0} \in \mathscr{G}_{0}$ such that $J\left(r_{0}\right)$ contains at least two distinct points, say $a^{\prime}, a^{\prime \prime}$. Note that $r_{0} \mid K \notin \mathscr{P}_{n, K}$. Indeed, assuming $r_{0} \mid K \in$ $\mathscr{P}_{n, K}$, we would find a polynomial $s_{0} \in \mathscr{P}_{n}$ such that $r_{0}\left|K=s_{0}\right| K$. Then we would have $a\left(r_{0}, q\right)=a\left(s_{0}, q\right)$ and $b\left(r_{0}, q\right)=b\left(s_{0}, q\right)$ for all $q \in \mathscr{Q}_{n}(\Omega)$ with $s_{0} \in \mathscr{P}_{n, q}$. But $a\left(s_{0}, q\right)=b\left(s_{0}, q\right)=L_{\gamma}\left(s_{0}\right)$ whenever $s_{0} \in \mathscr{P}_{n, q}$. This would imply $J\left(r_{0}\right)$ singleton or empty, which contradictis our hypothesis. Therefore, $\mathscr{P}_{n, K}+\mathrm{R} r_{0} \neq \mathscr{P}_{n, K}$, where we write $r_{0}$ for $r_{0} \mid K$.

We may define on $\mathscr{P}_{n, K}+\mathrm{R} r_{0}$ two linear functionals $\phi^{\prime}\left(p+\lambda r_{0}\right)=\phi(p)+$ $\lambda a^{\prime}$ and $\phi^{\prime \prime}\left(p+\lambda r_{0}\right)=\phi(p)+\lambda a^{\prime \prime}$, for all $p \in \mathscr{P}_{n, K}$ and $\lambda \in \mathrm{R}$. Clearly, $\phi^{\prime}, \phi^{\prime \prime}$ are two distinct extensions or $\phi$.

If $\phi_{q}^{\prime}=\phi^{\prime}\left|\mathscr{P}_{n, q}+\mathrm{R} r_{0}, \phi_{q}^{\prime \prime}=\phi^{\prime \prime}\right| \mathscr{P}_{n, q}+\mathrm{R} r_{0}$, we have $\left\|\phi_{q}^{\prime}\right\|=\left\|\phi_{q}^{\prime \prime}\right\|=$ $\left\|\phi_{q}\right\|=\phi\left(q^{-1}\right)>0$, since $a^{\prime}, a^{\prime \prime} \in J_{q}\left(r_{0}\right)$, and so $\phi_{q}^{\prime}$, $\phi_{q}^{\prime \prime}$ are Hahn-Banach extensions of $\phi_{q}$, for every $q \in \mathscr{Q}_{n}(\Omega)$.

We will apply Theorem 3.7 to both $\phi^{\prime}, \phi^{\prime \prime}$. The set $\mathscr{Q}_{n}(\Omega)$ has a partial ordering defined in the proof of Theorem 4.2. The set of the zeros in $\Omega$ of $q_{(1, \ldots, 1)}$ contains the set of zeros in $\Omega$ of every function $q \in \mathscr{Q}_{n}(\Omega)$. Putting $\mathscr{F}_{q}=$ $\mathscr{P}_{n, q}+\mathrm{R} r_{0} \subset C_{\mathrm{R}}(\Omega) / q$ (because $r_{0} \in C_{\mathrm{R}}(\Omega)$ ), we obviously have $1, q^{-1} \in$ $\mathscr{F}_{q}$, and $\mathscr{F}_{q^{\prime}} \subset \mathscr{F}_{q^{\prime \prime}}$, whenever $q^{\prime} \uparrow q^{\prime \prime}$. Therefore, we may apply Theorem 3.7 to the subspace $\mathscr{F}=\sum_{q \in \mathscr{Q}_{n}(\Omega)} \mathscr{F}_{q}=\mathscr{P}_{n, K}+\mathrm{R} r_{0}$ of $\sum_{q \in \mathscr{Q}_{n}(\Omega)} C_{\mathrm{R}}(\Omega) / q=$ $C_{\mathrm{R}}(\Omega) / \mathscr{Q}_{n}(\Omega)$, and to the functionals $\phi^{\prime}, \phi^{\prime \prime}$, which extend to two positive functionals $\psi^{\prime}, \psi^{\prime \prime}$ on $C_{\mathrm{R}}(\Omega) / \mathscr{Q}_{n}(\Omega)$. Clearly, $\psi^{\prime}, \psi^{\prime \prime}$ are distinct, since $\phi^{\prime}, \phi^{\prime \prime}$ are so. But the functionals $\psi^{\prime}, \psi^{\prime \prime}$ have the same representing measure, because the representing measure of $\gamma$ is supposed to be unique. This is a contradiction, showing that $J(r)$ must be singleton for all $r \in \mathscr{G}_{0}$. 
As for the last assertion, setting $a(r)=\sup _{q \in \mathscr{Q}_{n}(\Omega)} a(r, q), b(r)=\inf _{q \in \mathscr{Q}_{n}(\Omega)}$ $b(r, q)$, it is easily seen that $J(r)=[a(r), b(r)]$ for an $r \in \mathscr{G}_{0}$ whenever $J(r)$ is nonempty, which clearly implies our claim.

REMARK. Let $\gamma=\left(\gamma_{\alpha}\right)_{\alpha \in \mathrm{Z}_{+}^{n}}$ be a moment sequence on $\mathbf{R}^{n}$. As before, for all $r \in \mathscr{G}_{0}$ and $\alpha \in \mathrm{Z}_{+}^{n}$, we define the quantities

$$
\begin{aligned}
& a(r, \alpha)=\sup _{p \in \mathscr{P}_{n, \alpha}}\left[-L_{\gamma}(p)-L_{\gamma}\left(p_{\alpha}\right)\left\|q_{\alpha}(p+r)\right\|_{\infty}\right], \\
& b(r, \alpha)=\inf _{p \in \mathscr{P}_{n, \alpha}}\left[L_{\gamma}\left(p_{\alpha}\right)\left\|q_{\alpha}(p+r)\right\|_{\infty}-L_{\gamma}(p)\right] .
\end{aligned}
$$

We have $a(r, \alpha) \leq b(r, \alpha)$ for all $\alpha \in \mathrm{Z}_{+}^{n}$, and we can construct the interval $J_{\alpha}(r)=[a(r, \alpha), b(r, \alpha)]$, as well as the set $J(r)=\cap_{\alpha \in Z_{+}^{n}} J_{\alpha}(r)$. Theorem 4.5 asserts, for this special case, that the representing measure of $\gamma$ is uniquely determined if and only if

$$
\sup _{\alpha \in \mathbf{Z}_{+}^{n}} a(r, \alpha)=\inf _{\alpha \in \mathbb{Z}_{+}^{n}} b(r, \alpha),
$$

for all $r \in \mathscr{G}_{0}$.

In other words, if $\mu$ is a positive measure on $R^{n}$ having moments of all orders, this measure is uniquely determined by its moments if and only if

$$
\begin{aligned}
\sup _{\alpha \in \mathbb{Z}_{+}^{n}} \sup _{p \in \mathscr{P}_{n, \alpha}}\left[-\int\left(p+\left\|q_{\alpha}(p+r)\right\|_{\infty} p_{\alpha}\right) d \mu\right] \\
=\inf _{\alpha \in \mathbb{Z}_{+}^{n}} \inf _{p \in \mathscr{P}_{n, \alpha}}\left[\int\left(\left\|q_{\alpha}(p+r)\right\|_{\infty} p_{\alpha}-p\right) d \mu\right],
\end{aligned}
$$

for all $r \in \mathscr{G}_{0}$

Theorem 4.5 characterizes the uniqueness of the representing measure of a moment sequence in numerical terms. For other uniqueness results in a similar spirit, see also [10] and [15]. The next results deal with the uniqueness in terms of density (see Proposition 4.7). For the sake of simplicity, we restrict ourselves to the case $K=\mathrm{R}^{n}$, although this hypothesis in not essential.

Let $\mathscr{R}_{n}$ be the algebra of all rational functions on $\mathrm{R}^{n}$, with denominators in the set $\left\{q^{-1} \in \mathscr{P}_{n} ; q \in \mathscr{Q}_{n}\right\}$. Obviously, $\mathscr{R}_{n} \subset C_{\mathrm{R}}\left(\left(\mathrm{R}_{\infty}\right)^{n}\right) / \mathscr{Q}_{n}$. Let also

$$
\mathscr{R}_{n, \alpha}=\left\{r \in \mathscr{R}_{n} ; r q_{\alpha} \in C_{\mathrm{R}}\left(\left(\mathrm{R}_{\infty}\right)^{n}\right)\right\}=\mathscr{R}_{n} \cap C_{\mathrm{R}}\left(\left(\mathrm{R}_{\infty}\right)^{n}\right) / q_{\alpha} .
$$

We clearly have $\mathscr{P}_{n, \alpha} \subset \mathscr{R}_{n, \alpha}$ and $\mathscr{R}_{n, \alpha} \subset \mathscr{R}_{n, \beta}$ for all $\alpha, \beta \in \mathrm{Z}_{+}^{n}$ with $\alpha \prec \beta$.

Set $\mathscr{R}_{0}=\mathscr{R}_{n} \cap C_{\mathrm{R}}\left(\left(\mathrm{R}_{\infty}\right)^{n}\right)$, which is an algebra of rational continuous functions, dense in $C_{\mathrm{R}}\left(\left(\mathrm{R}_{\infty}\right)^{n}\right)$ (see the proof of Theorem 4.2). 
Proposition 4.6. An n-sequence $\gamma=\left(\gamma_{\alpha}\right)_{\alpha \in \mathrm{Z}_{+}^{n}}\left(\gamma_{0}>0\right)$ of real numbers is a moment sequence on $\mathbf{R}^{n}$ if and only if the linear functional $L_{\gamma}: \mathscr{P}_{n} \rightarrow \mathbf{R}$ has the properties $L_{\gamma}\left(p_{\alpha}\right)>0, \alpha \in Z_{+}^{n}$, and for every $p \in \mathscr{P}_{n}$ written as $p=\sum_{k \in K} p_{\alpha_{k}} r_{k}$, where $r_{k} \in \mathscr{R}_{0}$ for all $k \in K, K \subset \mathbf{Z}_{+}$finite, we have $\left|L_{\gamma}(p)\right| \leq \sum_{k \in K} L_{\gamma}\left(p_{\alpha_{k}}\right)\left\|r_{k}\right\|_{\infty}$.

Proof. Note that $\mathscr{R}_{n}=\sum_{\alpha \in \mathrm{Z}_{+}^{n}} p_{\alpha} \mathscr{R}_{0}$. Set

$$
W=\operatorname{co}\left(\bigcup_{\alpha \in Z_{+}^{n}}\left\{r \in p_{\alpha} \mathscr{R}_{0} ;\left\|q_{\alpha} r\right\|_{\infty} \leq L_{\gamma}\left(p_{\alpha}\right)^{-1}\right\}\right) \text {. }
$$

If we put

$$
\stackrel{\sigma_{\gamma}(f)}{=} \inf \left\{\sum_{\alpha \in K} L_{\gamma}\left(p_{\alpha}\right)\left\|r_{\alpha}\right\|_{\infty} ; f=\sum_{\alpha \in K} q_{\alpha}^{-1} r_{\alpha}, r_{\alpha} \in \mathscr{R}_{0}, K \subset Z_{+}^{n}, K \text { finite }\right\} .
$$

for all $f \in \mathscr{R}_{n}$, we have $\sigma_{\gamma}=\sigma_{W}$, via Remark 3.5(2). Therefore, the condition from the statement is equivalent to the estimate $\left|L_{\gamma}(p)\right| \leq \sigma_{W}(p), p \in \mathscr{P}_{n}$. This implies that $\left\|L_{\gamma} \mid \mathscr{P}_{n, \alpha}\right\|=L_{\gamma}\left(p_{\alpha}\right), \alpha \in Z_{+}^{n}$, via Lemma 2.2. Therefore, if $L_{\gamma}: \mathscr{P}_{n} \rightarrow \mathrm{R}$ satisfies the conditions from the statement, then $\gamma$ is a moment sequence, by Theorem 4.1.

Conversely, if $\gamma$ is a moment sequence and $\mu$ is a representing measure of $\gamma$, choosing a $p \in \mathscr{P}_{n}$ such that $p=\sum_{k \in K} p_{\alpha_{k}} r_{k}$, with $r_{k} \in \mathscr{R}_{0}, k \in K \subset \mathbf{Z}_{+}$ finite, we have

$$
\left|L_{\gamma}(p)\right| \leq \int|p(t)| d \mu(t) \leq \sum_{k \in K} L_{\gamma}\left(p_{\alpha_{k}}\right)\left\|r_{k}\right\|_{\infty} .
$$

In the next statement we keep the notation from Proposition 4.6 and its proof. We show that the representing measure for the sequence $\gamma$ is uniquely determined if and only if $\mathscr{P}_{n}$ is "dense" in $\mathscr{R}_{n}$ with respect to the seminorm $\sigma_{W}(r), r \in \mathscr{R}_{n}$. More precisely, we have the following.

Proposition 4.7. Suppose that the functional $L_{\gamma}$ has the properties $L_{\gamma}\left(p_{\alpha}\right)>0, \alpha \in Z_{+}^{n}$, and $\left|L_{\gamma}(p)\right| \leq \sigma_{\gamma}(p), p \in \mathscr{P}_{n}$. A representing measure for the sequence $\gamma$ is uniquely determined if and only if the normed space $\left(\mathscr{P}_{n}+\mathscr{Z}\right) / \mathscr{Z}$ is dense in the normed space $\mathscr{R}_{n} / \mathscr{Z}$, where $\mathscr{Z}=\operatorname{ker}\left(\sigma_{\gamma}\right)$.

Proof. The existence of a representing measure for $\gamma$ follows from the previous result. According to Remark 2.3, the uniqueness of the extension of the functional $L_{\gamma}$ to $\mathscr{R}_{n}$ is characterized by the condition in the statement. Note that $\mathscr{R}_{0} \subset \mathscr{R}_{n, \alpha}$ for all $\alpha \in \mathrm{Z}_{+}^{n}$. Moreover, as noticed before, $\mathscr{R}_{0}$ is dense 
in $C_{\mathrm{R}}\left(\left(\mathrm{R}_{\infty}\right)^{n}\right)$. Assuming the density from the statement, let $\phi$ be the unique extension of $L_{\gamma}$ to $\mathscr{R}_{n}$. Let $\phi_{\alpha}=\phi \mid \mathscr{R}_{n, \alpha}$ for all $\alpha \in \mathrm{Z}_{+}^{n}$. The density of $\mathscr{R}_{0}$ in $C_{\mathrm{R}}\left(\left(\mathrm{R}_{\infty}\right)^{n}\right)$ implies the density of $\mathscr{R}_{n, \alpha}$ in the normed space $C_{\mathrm{R}}\left(\left(\mathrm{R}_{\infty}\right)^{n}\right) / q_{\alpha}$ for all $\alpha \in \mathrm{Z}_{+}^{n}$. Therefore, every functional $\phi_{\alpha}$ extends uniquely to a functional $\psi_{\alpha}$ on $C_{\mathrm{R}}\left(\left(\mathrm{R}_{\infty}\right)^{n}\right) / q_{\alpha}$, having the same norm. Moreover, if $\alpha \prec \beta$, we have $\psi_{\alpha}=\psi_{\beta} \mid C_{\mathrm{R}}\left(\left(\mathrm{R}_{\infty}\right)^{n}\right) / q_{\alpha}$, because of the estimate $\|f\|_{\infty, \beta} \leq\|f\|_{\infty, \alpha}$, for all $f \in C_{\mathrm{R}}\left(\left(\mathrm{R}_{\infty}\right)^{n}\right) / q_{\alpha}$ and $\alpha, \beta \in \mathrm{Z}_{+}^{n}$. Consequently, we have a well-defined linear functional $\phi: C_{\mathrm{R}}\left(\left(\mathrm{R}_{\infty}\right)^{n}\right) / \mathscr{Q}_{n} \rightarrow \mathrm{R}$, which is continuous, since $\psi_{\alpha}=$ $\psi \mid C_{\mathrm{R}}\left(\left(\mathbf{R}_{\infty}\right)^{n}\right) / q_{\alpha}$ is continuous for all $\alpha \in \mathrm{Z}_{+}^{n}$. The form $\psi$ is positive, by Theorem 3.2. The uniqueness of $\psi$ is equivalent to the uniqueness of the representing measure for the sequence $\gamma$, again by Theorem 3.2.

REMARK. The density in the previous statement means that for every $r \in \mathscr{R}_{0}$ we can find a sequence $\left(p_{k}\right)_{k}$ in $\mathscr{P}_{n}$ such that $\lim _{k \rightarrow \infty} \sigma_{\gamma}\left(r-p_{k}\right)=0$.

An alternate discussion, in the spirit of [9], leads to assertions parallel to Theorems 4.2 and 4.5.

Let $R_{\infty}^{n}=R^{n} \cup\{\infty\}$ be the one point compactification of the Euclidean space $\mathrm{R}^{n}$, for a fixed integer $n \geq 1$. We consider the family $\mathscr{Q}$ consisting of all rational functions of the form $q_{k}(t)=\left(1+\|t\|^{2}\right)^{-k}, t=\left(t_{1}, \ldots, t_{n}\right) \in \mathrm{R}^{n}$, with $\|t\|^{2}=t_{1}^{2}+\cdots+t_{n}^{2}$, and $k \in \mathbf{Z}_{+}$arbitrary. The functions $q_{k}$ may be extended with zero at the point $\infty$. In this way, $\mathscr{Q}$ becomes a multiplicative family in $C_{\mathrm{R}}\left(\mathrm{R}_{\infty}^{n}\right)$.

As before, $\mathscr{P}_{n}$ is the algebra of all polynomial functions on $\mathrm{R}^{n}$, with real coefficients. Let also $\mathscr{P}_{n, k}=\left\{p \in \mathscr{P}_{n}, \lim _{t \rightarrow \infty} q_{k}(t) p(t)\right.$ exists $\}, k \in \mathbf{Z}_{+}$.

We restrict our discussion to the case of moment problems on $\mathrm{R}^{n}$, although we can state and prove the next theorem, with minor changes, on an arbitrary (unbounded) closed set in $\mathrm{R}^{n}$.

THEOREM 4.8. An n-sequence $\gamma=\left(\gamma_{\alpha}\right)_{\alpha \in \mathrm{Z}_{+}^{n}}\left(\gamma_{0}>0\right)$ of real numbers is a moment sequence on $\mathrm{R}^{n}$ if and only if the linear functional $L_{\gamma}: \mathscr{P}_{n} \rightarrow \mathrm{R}$ has the properties $L_{\gamma}\left(p_{k}\right)>0$ and

$$
\left|L_{\gamma}(p)\right| \leq L_{\gamma}\left(p_{k}\right) \sup _{t \in \mathrm{R}^{n}}\left|q_{k}(t) p(t)\right|, \quad p \in \mathscr{P}_{n, k}, k \geq 0 .
$$

Proof. As for the proof of Theorem 4.2, the stated conditions are necessary, as one can easily see.

Conversely, we will apply Theorem 3.7 to the functional $\phi=L_{\gamma}$.

Note that the space $C_{\mathrm{R}}\left(\mathrm{R}_{\infty}^{n}\right)$ is separable because the algebra $C_{\mathrm{R}}\left(\mathrm{R}_{\infty}^{n}\right)$ is generated by the family $\left\{1, q_{1}(t), t_{1} q_{1}(t), \ldots, t_{n} q_{1}(t)\right\}$, which separates the points of $\mathbf{R}_{\infty}^{n}$, via the Weierstrass-Stone density theorem. Note also that $q_{1}(\omega)=0$ if and only if $\omega=\infty$ and if and only if $q_{k}(\omega)=0, k \geq 1$. Moreover, it is easily 
seen that $q_{k} \mid q_{m}$ in $\mathscr{Q}$ if and only if $k \leq m$ for all integres $k, m \geq 1$, and so we have a total ordering on the set 2 .

We clearly have $1, p_{k} \in \mathscr{P}_{n, k}$ and $\mathscr{P}_{n, k} \subset \mathscr{P}_{n, m}$ whenever $k \leq m$, for all $k, m \geq 1$. This discussion shows that the conditions to apply Theorem 3.7 are fulfilled.

If $\phi_{k}=\phi \mid \mathscr{P}_{n, k}$, the hypothesis implies the estimates $\left\|\phi_{k}\right\| \leq L_{\gamma}\left(p_{k}\right)$, $k \geq 0$. As $p_{k}$ is a norm one element, the conditions from the statement imply the conditions $\left\|\phi_{k}\right\|=L_{\gamma}\left(p_{k}\right)>0, k \geq 0$, which, in turn, imply the existence of a positive extension $\psi$ of $\phi$ to $C_{\mathrm{R}}\left(\mathrm{R}_{\infty}^{n}\right) / \mathscr{Q}$, by Theorem 3.7. This shows the existence of a positive extension $\psi$ of $\phi$, which is given by a representing measure whose support can be chosen in $\mathrm{R}^{n}$, by Remark 3.8(1), and which is, in particular, a representing measure for $\gamma$.

Remarks 4.9. (1) An alternate proof of Theorem 4.8 can be obtained via Theorem 4 from [14]. On the other hand, the full force of Theorem 3.7 is needed for the proof of Theorem 4.2 (or Theorem 4.1), because the set $\left(\mathrm{R}_{\infty}\right)^{n} \backslash \mathrm{R}^{n}$ contains more than one point. As a matter of fact, we think that the direct application of Theorem 4 from [14] does not suffice the get Theorem 4.2.

(2) The use of the simpler spaces $\mathscr{P}_{n, \alpha}$ in Theorem 4.1, rather than that of $\mathscr{P}_{n, k}$ in Theorem 4.8, makes the former more appropriate for the calculations of the sup-norms in their statement (see Remarks 4.11 and 4.12).

Our approach to the moment problem in unbounded sets shows that it can be regarded as a "singular moment problems", because the moments are defined via singular functions (with respect to the given domain of definition). The same methods can be applied in other cases, where the singularities appear in an obvious manner.

Example 4.10. Let $\mathrm{S}_{n}$ be the compact set $\left\{t=\left(t_{1}, \ldots, t_{n}\right) \in \mathrm{R}^{n} ; t_{1}^{2} \leq\right.$ $\left.1, \ldots, t_{n}^{2} \leq 1\right\}$. In the space $C_{\mathrm{R}}\left(\mathrm{S}_{n}\right)$, we consider the multiplicative family $\mathscr{Q}=\left\{q_{\alpha} ; \alpha \in \mathrm{Z}_{+}^{n}\right\}$, where $q_{\alpha}(t)=t^{2 \alpha}, t \in \mathbf{S}_{n}$.

Let $\mathscr{S}_{\alpha}=\left\{p \mid \mathrm{S}_{n} ; p \in \mathscr{P}_{n, \alpha}\right\}$, let $\mathscr{F}_{\alpha}=\mathscr{S}_{\alpha} / q_{\alpha}$, and let $\mathscr{F}=\sum_{\alpha} \mathscr{F}_{\alpha}$.

Given $L: \mathscr{F} \rightarrow \mathrm{R}$ a linear functional with $L(1)>0$, we set $\delta_{\alpha}=L\left(q_{\alpha}^{-1}\right)$, $\alpha \in \mathrm{Z}_{+}^{n}$. According to Theorem 3.7, the functional $L$ has a positive extension to $C_{\mathrm{R}}\left(\mathrm{S}_{n}\right) / \mathscr{Q} \rightarrow \mathrm{R}$ if and only if $\delta_{\alpha}>0$ and

$$
|L(f)| \leq \delta_{\alpha}\left\|q_{\alpha} f\right\|_{\infty}, \quad f \in \mathscr{F}_{\alpha},
$$

for all $\alpha \in \mathrm{Z}_{+}^{n}$.

In the affirmative case, we can find a positive measure $\mu$ with support in $\mathrm{S}_{n} \backslash\left\{t ; \min \left\{t_{1}^{2}, \ldots, t_{n}^{2}\right\}=0\right\}$, such that $\delta_{\alpha}=\int q_{\alpha}^{-1} d \mu, \alpha \in \mathbf{Z}_{+}^{n}$. 
REMARK 4.11. The sup-norms $\left\|q_{\alpha} p\right\|_{\infty}=\sup _{t \in \mathrm{R}^{n}}\left|q_{\alpha}(t) p(t)\right|, p \in \mathscr{P}_{n, \alpha}$, $\alpha \in \mathrm{Z}_{+}^{n}$, which occur in the statement of Theorem 4.1 , can be computed in a fairly explicit manner. We briefly indicate a method of computation.

First of all, we identify the space $\left(\mathrm{R}_{\infty}\right)^{n}$ with the set $\mathrm{T}^{n} \subset \mathrm{C}^{n}$, where $\mathrm{T}$ is the unit circle in the complex plane, via the map

$\left(\mathrm{R}_{\infty}\right)^{n} \ni t=\left(t_{1}, \ldots, t_{n}\right) \rightarrow\left(\frac{1-t_{1}^{2}}{1+t_{1}^{2}}+i \frac{2 t_{1}}{1+t_{1}^{2}}, \ldots, \frac{1-t_{n}^{2}}{1+t_{n}^{2}}+i \frac{2 t_{n}}{1+t_{n}^{2}}\right) \in \mathrm{T}^{n}$

which is a homeomorphism (the point $\infty \in \mathrm{R}_{\infty}$ is mapped into $-1 \in \mathrm{T}$ ). If $\Phi: \mathrm{T}^{n} \rightarrow\left(\mathrm{R}_{\infty}\right)^{n}$ is the inverse of this homeomorphism, we set $\hat{p}_{\alpha}=\left(q_{\alpha} p\right) \circ \Phi$ for all $p \in \mathscr{P}_{n, \alpha}, \alpha \in \mathrm{Z}_{+}^{n}$. It is clear that $\left\|q_{\alpha} p\right\|_{\infty}=\sup _{\zeta \in \mathrm{T}^{n}}\left|\hat{p}_{\alpha}(\zeta)\right|=\left\|\hat{p}_{\alpha}\right\|_{\infty}$ for all $p \in \mathscr{P}_{n, \alpha}, \alpha \in \mathrm{Z}_{+}^{n}$, which reduces our problem to the computation of some sup-norms on $\mathrm{T}^{n}$. Setting

$$
z=\frac{1-u^{2}}{1+u^{2}}+i \frac{2 u}{1+u^{2}} \in \mathrm{T}, \quad u \in \mathrm{R},
$$

for all nonnegative integers $k, m$, we have the identities

$$
\frac{u^{2 k}}{\left(1+u^{2}\right)^{m}}=\frac{1}{2^{m}}\left(1+\frac{z+\bar{z}}{2}\right)^{m-k}\left(1-\frac{z+\bar{z}}{2}\right)^{k}
$$

if $2 k \leq 2 m$, and

$$
\frac{u^{2 k+1}}{\left(1+u^{2}\right)^{m}}=\frac{1}{2^{m}}\left(\frac{z-\bar{z}}{2 i}\right)\left(1+\frac{z+\bar{z}}{2}\right)^{m-1-k}\left(1-\frac{z+\bar{z}}{2}\right)^{k}
$$

if $2 k+1 \leq 2 m$. These identities show that the function $\hat{p}_{\alpha}$ is a real-valued trigonometric polynomial, whose coefficients can be obtained from those of $p$, for all $p \in \mathscr{P}_{n, \alpha}, \alpha \in \mathbf{Z}_{+}^{n}$.

Let $\lambda_{n}$ be the normalized Lebesgue measure on $\mathrm{T}^{n}$, and let $L^{2}\left(\mathrm{~T}^{n}\right)=$ $L^{2}\left(\mathrm{~T}^{n}, \lambda_{n}\right)$ be the Hilbert space of all measurable and square integrable functions on $\mathrm{T}^{n}$. For all $p \in \mathscr{P}_{n, \alpha}, \alpha \in \mathrm{Z}_{+}^{n}$, we denote by $T_{p, \alpha}$ the multiplication operator $T_{p, \alpha} f(\zeta)=\hat{p}_{\alpha}(\zeta) f(\zeta), \zeta \in \mathrm{T}^{n}, f \in L^{2}\left(\mathrm{~T}^{n}\right)$, which is self-adjoint, because $\hat{p}_{\alpha}$ is a real valued function. It is well known, and easily seen, that $\left\|T_{p, \alpha}\right\|=\left\|\hat{p}_{\alpha}\right\|_{\infty}$. Moreover, as $-\left\|T_{p, \alpha}\right\| \leq T_{p, \alpha} \leq\left\|T_{p, \alpha}\right\|$, we infer the formula

$$
\left\|\hat{p}_{\alpha}\right\|_{\infty}=\inf \left\{M \geq 0 ; M \pm T_{p, \alpha} \geq 0\right\}
$$

for all $p \in \mathscr{P}_{n, \alpha}, \alpha \in \mathbf{Z}_{+}^{n}$.

We can give the formula above a more classical flavor. If the function $\hat{p}_{\alpha}$ is written as $\hat{p}_{\alpha}(\zeta)=\sum_{\tau \in Z^{n}} a_{\tau} \zeta^{\tau}$ (where $\zeta=\left(\zeta_{1}, \ldots, \zeta_{n}\right) \in \mathrm{T}^{n}$ and $\zeta^{\tau}=$ 
$\zeta_{1}^{\tau_{1}} \cdots \zeta_{n}^{\tau_{n}}$ for all $\left.\tau=\left(\tau_{1}, \ldots, \tau_{n}\right) \in \mathbf{Z}^{n}\right)$, we have $\bar{a}_{\tau}=a_{-\tau}$ and $a_{\tau} \neq 0$ only for a finite number of indices. In fact, if $p(t)=\sum_{\xi<2 \alpha} d_{\xi} t^{\xi}$, and thus $q_{\alpha} p=\sum_{\xi<2 \alpha} d_{\xi} r_{\xi, \alpha}$, with $r_{\xi, \alpha}$ as in Theorem 4.5, and using the orthonormality of the family $\left(\zeta^{\kappa}\right)_{\kappa \in Z^{n}}$ in $L^{2}\left(\mathrm{~T}^{n}\right)$, we obtain $a_{\tau}=\sum_{\xi<2 \alpha} d_{\xi}\left\langle r_{\xi, \alpha} \circ \Phi, \zeta^{\tau}\right\rangle$ for all indices $\tau$. Obviously, the scalar products $\left\langle r_{\xi, \alpha} \circ \Phi, \zeta^{\tau}\right\rangle$ are calculable and allow us to express the coefficients $\left(a_{\tau}\right)$ as linear combinations of the coefficients $\left(d_{\xi}\right)$.

Let $f(\zeta)=\sum_{\kappa \in Z^{n}} c_{\kappa} \zeta^{\kappa}$ be arbitrary in $L^{2}\left(\mathrm{~T}^{n}\right)$. By reasons of density, we may also assume that $c_{\kappa} \neq 0$ only for a finite number of indices. We infer easily that

$$
\left\langle\hat{p}_{\alpha} f, f\right\rangle=\sum_{\kappa \in Z^{n}}\left(\sum_{\tau \in Z^{n}} a_{\tau} c_{\kappa-\tau}\right) \bar{c}_{\kappa} .
$$

Therefore, the formula above expressing the sup-norm of $\hat{p}_{\alpha}$ can be written as

$$
\left\|\hat{p}_{\alpha}\right\|_{\infty}=\inf \left\{M \geq 0 ; \sum_{\kappa \in Z^{n}}\left(M c_{\kappa} \pm \sum_{\tau \in Z^{n}} a_{\tau} c_{\kappa-\tau}\right) \bar{c}_{\kappa} \geq 0,\left(c_{\kappa}\right)_{\kappa} \in \mathrm{C}^{(\infty)}\right\},
$$

where $\mathrm{C}^{(\infty)}$ stands for the set of all sequences of complex numbers $\left(c_{\kappa}\right)_{\kappa \in Z^{n}}$, having finite support.

Remark 4.12. The use of the space $\mathrm{R}_{\infty}^{n}$ for the calculation of the supnorms in Theorem 4.8 seems to be more intricate. For instance, the standard embedding of $\mathbf{R}^{n}$ into the unit sphere of $\mathbf{R}^{(n+1)^{2}}$, given by

$$
\mathrm{R}^{n} \ni t=\left(t_{1}, \ldots, t_{n}\right) \rightarrow\left(\frac{t_{j} t_{k}}{1+\|t\|^{2}}\right)_{j, k=0}^{n} \in \mathbf{R}^{(n+1)^{2}},
$$

where $t_{0}=1$, cannot be extended continuously at $\infty$, if $n>1$.

\section{REFERENCES}

1. Akhiezer, N. I., The Classical Moment Problem and some related Questions in Analysis, Oliver and Boyd, Edinburgh, 1965.

2. Berg, C., Christensen, J. P. R., Ressel, P., Harmonic Analysis on Semigroups, Springer-Verlag, New York/Berlin/Heidelberg/Tokyo, 1984.

3. Curto, R. E., and Fialkow, L. A., Solution of the truncated complex momentnt problem for flat data, Mem. Amer. Math. Soc. 568 (1996).

4. Curto, R. E., and Fialkow, L. A., Flat extensions of positive moment matrices: Recursively generated relations, Mem. Amer. Math. Soc. 648 (1998).

5. Dunford, N., and Schwartz, J. T., Linear Operators, Part I", Interscience Publishers, New York, London, 1958.

6. Fuglede, B., The multidimensional moment problem, Exposition. Math. 1 (1983), 47-65. 
7. Haviland, E. K., On the momentum problem for distribution functions in more than one dimension, Amer. J. Math. 57 (1935), 562-568; ibidem II, Amer. J. Math. 58, 164-168.

8. Megginson, R. E., An Introduction to Banach Space Theory, Springer-Verlag, New York, 1998.

9. Putinar, M., and Vasilescu, F.-H., Solving moment problems by dimensional extension, Ann. of Math. 149 (1999), 1087-1107.

10. Putinar, M., and Vasilescu, F.-H., A uniqueness criterion in the multivariate moment problem, Math. Scand. 92 (2003), 295-300.

11. Robertson, A. P., and Robertson, W. J., Topological Vector Spaces.

12. Schmüdgen, K., Unbounded Operator Algebras and Representation Theory, Operator Theory: Advances and Applications Vol. 37, Birkhäuser Verlag, Basel/Boston/Berlin, 1990.

13. Shohat, J., and Tamarkin, J., The Problem of Moments, Math. Surveys Vol. 1, Amer. Math. Soc., Providence, RI, 1943.

14. Stochel, J., Solving the truncated moment problem solves the full moment problem, Glasgow Math. J. 43 (2001), 335-341.

15. Vasilescu, F.-H., Hamburger and Stieltjes moment problems in several variables, Trans. Amer. Math. Soc. 354 (2001), 1265-1278.

16. van der Waerden, B. L., Algebra, Vol. 2, Frederick Ungar Publ. Co., New York, 1970.

\author{
LABORATOIRE PAUL PAINLEVÉ \\ U.F.R. DE MATHÉMATIQUES \\ UNIVERSITÉ DE LILLE I \\ 59655 VILLENEUVE D'ASCQ \\ FRANCE \\ E-mail: fhvasil@math.univ-lille1.fr
}

